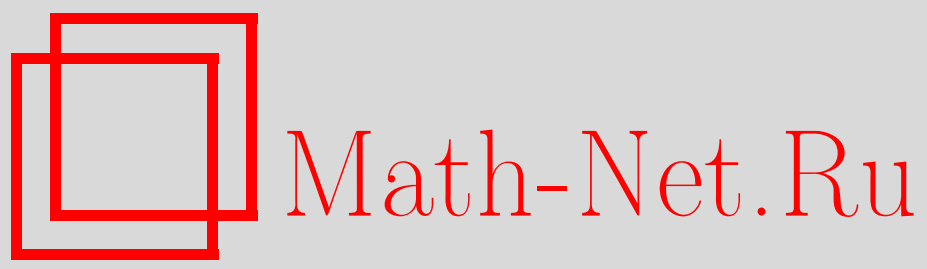

Н. Н. Яковлев, Е. А. Лукашев, Е. В. Радкевич, В. В. Палин, О парадигме внутренней турбулентности, Вестн. Сам. гос. техн. ун-та. Сер. Физ.мат. науки, 2015, номер 1, 155-185

DOI: https://doi.org/10.14498/vsgtu1418

Использование Общероссийского математического портала MathNet.Ru подразумевает, что вы прочитали и согласны с пользовательским соглашением

http://www . mathnet.ru/rus/agreement

Параметры загрузки:

IP : 52.23 .180 .231

26 апреля 2023 г., 17:49:11

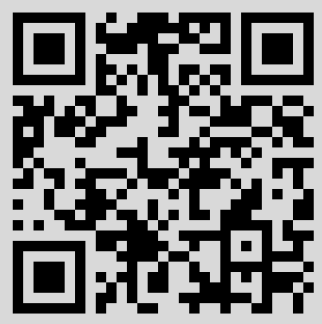




\title{
О ПАРАДИГМЕ ВНУТРЕННЕЙ ТУРБУЛЕНТНОСТИ
}

\author{
Н. Н. Яковлев ${ }^{1}$, Е. А. Лукашев ${ }^{1}$, \\ Е. В. Раджевич ${ }^{2}$ В. В. Палин ${ }^{2}$ \\ 1 OAO “Тураевское машиностроительное конструкторское бюро «Союз»" \\ Россия, 140080, Московская область, Лыткарино, промзона Тураево, 10. \\ 2 Московский государственный университет им. М. В. Ломоносова, \\ механико-математический факультет, \\ Россия, 119899, Москва, Воробьёвы горы.
}

\section{Аннотация}

Статья посвящена проблемам реконструкции начальной стадии внутренней турбулентности (без учета граничных эффектов). Введением вязкости и диффузионного расслоения проводится нестандартная регуляризация многокомпонентной системы Эйлера. Для нее получены аналог условия Гюгонио и аналог условия устойчивости Лакса. Исследована проблема локальной достижимости точек фазового пространства. Построены бифуркации однофронтовых решений усеченной системы Эйлера в двухфронтовые решения. Показан сверхзвуковой характер появления бифуркаций. Построена реконструкция начальной стадии внутренней турбулентности (без учета граничных эффектов), включая математическое описание возникновения двухскоростного режима (катастрофа Римана-Гюгонио) и перемежаемости.

Ключевые слова: реконструкция внутренней турбулентности, двухскоростной режим, катастрофа Римана-Гюгонио, перемежаемость, бифуркация, система Эйлера, кинетическое уравнение.

doi: http://dx.doi.org/10.14498/vsgtu1418

Введение. Существующие формы моделирования турбулентности отражают две крайности - волновую (вибрационную газовую динамику, см. например [1]) и наиболее распространенную диффузионную (статистическую) теорию турбулентности [2-5]. Современные подходы к теоретическому описанию начальной стадии внутренней турбулентности дают результаты, плохо согласуемые с экспериментом. Наша задача - построить реконструкцию начальной стадии турбулентности, промежуточную для этих двух крайностей и согласующую волновой и диффузионный характер турбулентности. В работах [6,7] был построен математический объект, реконструирующий основные

(C) 2015 Самарский государственный технический университет.

\section{Образец для цитирования}

Я к о в лев Н. Н., Лук аш ев Е. А., Р а дк е в и ч Е. В., Пал и н В. В. О парадигме внутренней турбулентности // Вестн. Сам. гос. техн. ун-та. Сер. Физ.-мат. науки, 2015. Т. 19, № 1. C. 155-185. doi: 10.14498/vsgtu1418.

\section{Сведения об авторах}

Николай Николаевич Яковлев (к.ф.-м.н.; amntksoyuz@mail.ru), генеральный директор. Евгений Алексеевич Лукашев (д.т.н., проф.; elukashov@yandex.ru), главный специалист. Евгений Владимирович Радкевич (д.ф.-м.н., проф; evrad07@gmail.com; автор, ведущий переписку), профессор, каф. дифференциальных уравнений.

Владимир Владимирович Палин (к.ф.-м.н.; grey_stranger84@mail.ru), ассистент, каф. дифференциальных уравнений. 
неустойчивости процесса и стабилизирующий их обратные связи. Грубо говоря, была построена аналоговая машина для воспроизводства базовых свойств турбулентности. Ее создание потребовало согласования микро- и макромасштабов, волнового и диффузионного процессов. В частности, удалось построить математическую реконструкцию начального этапа внутренней турбулентности, зарождение двухскоростного режима (катастрофы Римана-Гюгонио) и перемежаемости и предложить возможную гипотезу, объясняющую их зарождение.

Трудности расчетов таких процессов заключаются в том, что моделирование проводится одновременно на нескольких масштабных уровнях. K настоящему времени в экспериментальных исследованиях изучены многие детали начальной стадии процесса внутренней турбулентности, но общего теоретического представления об этом процессе пока не существует. Модель, используемая в данной работе, основана на многоскоростной системе уравнений Эйлера, распространение возмущений в которой приводит к катастрофе Римана-Гюгонио, являющейся причиной возникновения двухскоростного режима. Для описания образования флуктуаций плотностей, приводящих к перемежаемости, используется модифицированное уравнение Кана-Хилларда. Разномасштабность требует значительных вычислительных ресурсов. Двумерные расчеты основаны на явных и явно-итерационных алгоритмах, эффективно реализованных на многопроцессорной вычислительной системе.

Современный прогресс технологии, например в области материаловедения, связывают с созданием наноматериалов и технологий их производства нанотехнологий. Однако неверно утверждать, что об этом классе технологий не было известно до появления самого термина «нанотехнологии». K нанотехнологиям относят, в частности «золь-гель технологии», отработанные задолго до появления терминов «наноматериалы» и «нанотехнологии». Методические основы технологии производства дисперсионно упрочненных сплавов, которые по характерным структурным параметрам относятся к наноматериалам, начали закладываться академиком С. Т. Кишкиным начиная с работ 30-х годов [8]. В то же время появление самих терминов «наноматериалы» и «нанотехнологии», с одной стороны, поставило задачу - дать критерий, отличающий эти материалы и технологии от других. С другой стороны, эти термины поставили вопрос о характерных чертах, объединяющих эти материалы в единый класс. Ответ на эти вопросы заложен в самих терминах: характерный размер структуры больше микроразмера (размера атомной или молекулярной структуры), но гораздо меньше макроразмера образца материала (детали, конструкции, изделия). Это промежуточный размер - мезоскопический структурный уровень. Кроме того, введение данных терминов поставило вопрос об общих теоретических основах производства и эксплуатации этих материалов, т. е. потребовалось теоретическое обобщение. Как правило, «скелет» таких теоретических основ - математический аппарат, разработанный для решения специфических задач конкретной области. Понимание множества причин, от которых зависят требуемые свойства материала, заставляет при создании таких математических моделей учитывать многие факторы, определяющие процессы формирования структур жаропрочных материалов. Этого можно достичь, только используя совместно методы физического (экспериментального) и математического моделирования. Большая роль здесь отводится методам математического моделирования, которые развиваются бур- 
ными темпами благодаря использованию компьютерных технологий - быстродействующих компьютеров и высокоэффективных численных методов решения математических задач. В свою очередь, в процессе совершенствования математических моделей физических процессов их формулировка все больше смещается от построений, базирующихся на эмпирических или полуэмпирических зависимостях, к моделям, строящимся на физических закономерностях фундаментального характера, так называемых «первых принципах». При этом, естественно, происходит усложнение этих моделей, которое тем не менее оказывается оправданным благодаря, во-первых, развитию методов прикладной математики и компьютерной техники и, во-вторых, - значительной экономии времени и средств за счет снижения объема экспериментальных исследований, конструкторской проработки, стендовых и летных испытаний.

В данной работе представлена попытка обобщения термодинамической теории Кана-Хилларда на более широкий класс объектов по сравнению с тем, для которого она первоначально создавалась, - описание процесса спинодального распада сплава $[9,10]$.

Мезоструктурный масштаб представляет интерес не только для материаловедения. Характерный масштаб турбулентности (пульсации давления, завихренность по Тейлору и Томсону, моли Прандтля) также относится к этому структурному, но динамически подвижному уровню. К объектам этого же масштаба следует отнести характерные размеры фронта ударной волны и элементов ее структуры: скачков уплотнения, ударных, энтропийных и релаксационных слоев. Кроме того, в настоящее время наблюдается повышенный интерес к методам управления состоянием гиперзвуковых пограничных слоев, что связано с разработкой перспективных летательных аппаратов с гиперзвуковой крейсерской скоростью. Ламинарно-турбулентный переход приводит к существенному увеличению сопротивления и сильному локальному нагреву теплонагруженных поверхностей летательных аппаратов [11, 12]. Проблема увеличения области ламинарного режима обтекания является одной из важнейших при их проектировании. Все перечисленные системы обладают одним общим свойством: они могут быть охарактеризованы как неоднородные на промежуточном масштабном уровне. В связи с этим представляет интерес разработка математического аппарата, фиксирующего «вложенность» одного структурного уровня в другой. Попытка разработки математического аппарата с иерархической структурой «вложенности» подобного типа была предпринята нами на примере спинодального распада сплавов [12,13]; эти работы явились продолжением и развитием работ $[6,7]$, в частности, связанных с обобщением теории Кана-Хилларада на неизотермический случай.

1. Базовые свойства турбулентности. В этой статье мы продолжим исследование процессов с избыточной энергией, начатое в работах $[6,7,11,13]$. Кратко остановимся на выводах классической теории ударных волн [14].

[Течения жидкостей и газов, наблюдаемые в природных условиях и технических устройствах, делятся на два сильно отличающиеся друг от друга типа: ламинарные - спокойные, плавные, регулярные, и турбулентные, в которых скорость, давление, температура и другие гидродинамические величины изменяются хаотично, неупорядоченно не только во времени, но и в пространстве.

По-видимому, наиболее емкое и содержательное определение турбулентности принадлежит П. Брэдшоу (1971) [15]: «турбулентность - это трехмер- 
ное нестационарное движение, в котором вследствие растяжения вихрей создается непрерывное распределение пульсаций скорости в интервале длин волн от минимальных, определяемых вязкими силами, до максимальных, определяемых граничными условиями течения. Она является обычным состоянием движущейся жидкости, за исключением течений при малых числах Рейнольдса».

Существование резко различающихся ламинарных и турбулентных режимов течения было замечено еще в первой половине XIX в., но начало теории турбулентности было положено лишь в конце того же столетия в работах Осборна Рейнольдса (1883) [16,17]. Изучая условия перехода ламинарного течения жидкости в трубах в турбулентное, Рейнольдс установил существование общего критерия динамического подобия течений вязкой несжимаемой жидкости, названного впоследствии его именем: $\operatorname{Re}=U \cdot L / \nu$ - число Рейнольдса, где $U$ и $L$-характерные масштабы скорости и длины в рассматриваемом течении, а $\nu$ - кинематический коэффициент вязкости (например, для течения в трубе - $U$ - среднерасходная скорость, $L-$ диаметр трубы). Из опытов Рейнольдса следовало, что существенное влияние на характер течения в трубе оказывают возмущения на входе в нее. Если число Re достаточно мало, то эти возмущения затухают на некотором расстоянии, а течение в трубе является ламинарным. При достижении числом Re критического значения (в опытах Рейнольдса $\operatorname{Re}_{\text {кр }} \approx 2000$ ) возмущения не затухают и движение в трубе становится турбулентным.

Наиболее распространенной является интерпретация числа Рейнольдса как меры относительной значимости сил инерции и сил вязкости, действующих внутри жидкости. Силы инерции, если они существенно превосходят силы вязкости, что соответствует большим числам Re, вызывают перемешивание конечных обгемов жидкости, движущихся с разными скоростями. В результате осуществляется передача энергии от крупномасштабных структур (вихрей) к менее крупным, образующимся за счет потери устойчивости более крупных вихрей. Иными словами, крупномасштабные структуры играют роль аккумуляторов энергии из основного потока. Поглощая энергию основного потока, эти структуры оказываются сильно анизотропными, завихренными и существенно отличаются от течения к течению.

Основным механизмом генерации энергии турбулентности является деформация структур, представляющая собой трехмерный процесс, поэтому все развитые турбулентные течения являются трехмерными. Посредством нелинейных взаимодействий крупномасштабные структуры передают часть своей энергии менее крупным структурам и т. д., в результате реализуется так называемый каскадный механизм передачи энергии.

Впервые описание этого процесса было дано Льюисом Ричардсоном (1922) [16,17]. Согласно Ричардсону, каскадный процесс передачи энергии в турбулентном потоке, базирующийся на представлении о существовании иерархии вихрей, завершается на самых мелкомасштабных структурах вязкой диссипацией кинетической энергии в тепло. Однако понимание роли мелкомасштабной турбулентности в процессах турбулентного переноса пришло лишь после опубликования работы Джеффри Тэйлора (1935) [16,17], в которой впервые было введено понятие об однородной и изотропной турбулентности. Основным свойством такой турбулентности является ее слабая зависимость от индивидуальных особенностей течения (локальная изотропия). В предельном 
случае можно говорить об инвариантности свойств изотропной турбулентности относительно любых ортогональных преобразований (параллельных переносов, вращений и т. д.).. $]^{1}$

В общей картине гиперзвукового течения рассматривается зона, непосредственно примыкающая к ударной волне, где наблюдаются явления диссоциации и химических реакций, что позволило выделить основные факторы течения, визуализация которых есть предмет этой статьи, а именно:

1) двухскоростное течение (катастрофа Римана-Гюгонио);

2) образование флуктуаций плотности;

3) перемежаемость (полосчатость), на самой поверхности разрыва возникает рябь (аналог волны Маренгони);

4) согласование катастрофы Римана-Гюгонио и неустойчивостей, порождающих образование флуктуаций плотности;

5) при множественности стационарных состояний переходы по закону «все или ничего» (переходы ван-дер-ваальсовского типа);

6) характерные неустойчивости: срыв струй с фронта ударной волны (аналог тейлоровской неустойчивости) и образование вихрей.

Приведем экспериментальное обоснование сформулированных выше базовых факторов течения.

Во-первых, при рассмотрении гофрировочной неустойчивости ударных волн отмечается, что «произвольное возмущение течения складывается из энтропийно-вихревой волны и звуковой волны. [. . ] Возмущение в целом представляется линейной комбинацией возмущений обоих типов» [18, гл. ix, § 90, с. 472]. И далее [18, гл. ix, § 90, с. 476]: «К происхождению неустойчивости ударных волн [...] можно подойти [...], рассмотрев отражение от поверхности разрыва звука, падающего на нее со стороны сжатого газа. Поскольку ударная волна движется относительно газа впереди нее со сверхзвуковой скоростью, то в этот газ звук не проникает. В газе же позади волны будем иметь наряду с падающей звуковой волной еще и отраженную звуковую и энтропийно-вихревую волны (а на самой поверхности разрыва возникает рябь [(аналог

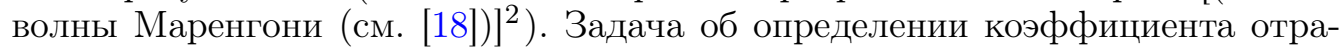
жения по своей постановке близка к задаче об исследовании устойчивости.»

Какой можно сделать вывод? На начальной стадии турбулентности образуется два типа возмущения с разными скоростями перемещения по газу (звуковые со скоростью звука и энтропийно-вихревые со скоростью потока газа), что позволяет выдвинуть гипотезу существования на мезоструктурном уровне двухскоростной гидродинамики, когда разные части газа (инертная часть и флуктуации уплотнений) обладают разными скоростями относительно неподвижного газа, разными коэффициентами переноса, если рассматривать поток этих флуктуаций, обусловленный градиентом их плотностей.

Как видим, эти факты дают экспериментальное обоснование пункта 1). Как отмечается Г. Николисом, И. Пригожиным [19, п. 8.4, с. 189], возникновение двухскоростного потока (двух типов возмущений с разными скоростями перемещения по газу) можно назвать катастрофой Римана-Гюгонио.

Далее, факт, что энтропийно-вихревые возмущения могут рассматривать-

\footnotetext{
${ }^{1}$ Текст в квадратных скобках цитирован по работе [14] (Google Scholar Cluster: $796228169334250115)$, выделение текста курсивом авторское.

${ }^{2}$ Вставка в квадратных скобках авторская.
} 
ся как флуктуации плотности, может быть обоснован соответствующим законом сохранения - законом сохранения циркуляции скорости (теорема Томпсона). Там же в [19, п. 8.4, с. 186] отмечается, что возникновение двухскоростного потока можно интерпретировать как «переходы [при множественных стационарных состояниях $]^{3}$ по закону "все или ничего" и теория катастроф». Эти факты дают экспериментальное обоснование пункта 5) о переходах вандер-ваальсовского типа.

Для пояснения напомним, что любое сложное пространственное распределение скорости можно представить в виде суперпозиции гармонических колебаний. В турбулентном потоке длина волны крупномасштабных колебаний сопоставима с характерным линейным размером задачи. Длина волны наиболее мелкомасштабных колебаний намного меньше характерных размеров задачи и, что самое главное, уменьшается по мере роста числа Рейнольдса. Поэтому в рассматриваемой суперпозиции (т. е. в спектре турбулентности) представлено очень большое число колебаний, длина волн которых сильно варьируется. Крупномасштабные колебания определяют энергию турбулентности, а мелкомасштабные - ее диссипацию, которая оказывается существенной при всех числах Рейнолдса (т. е. при любой сколь угодно малой вязкости).

Описанная структура потока характерна для многих задач гидродинамики. В теории турбулентности фундаментальную роль играют диссипация энергии $\langle\varepsilon\rangle$ и скалярная диссипация $\langle N\rangle$ :

$$
\begin{gathered}
\langle\varepsilon\rangle=\frac{1}{2} \nu \overline{\left(\partial_{x_{i}} u_{j}^{\prime}+\partial_{x_{j}} u_{i}^{\prime}\right)^{2}}, \quad\langle N\rangle=\overline{|\nabla z|^{2}} \\
\varepsilon=\frac{1}{2} \nu\left(\partial_{x_{i}} u_{j}^{\prime}+\partial_{x_{j}} u_{i}^{\prime}\right)^{2}=\nu|\omega|^{2}+2 \nu \partial_{x_{i}} u_{j}^{\prime} \partial_{x_{j}} u_{i}^{\prime}, \quad u^{\prime}=u-\bar{u} .
\end{gathered}
$$

Здесь $u_{i}$ - актуальная скорость; чертой сверху обозначено ее осредненное значение; штрихом - пульсационная скорость; $D, \nu$ - коэффициенты диффузии и кинематической вязкости; $x$-радиус вектор точки в декартовой прямоугольной системе координат; $\omega=\nabla \cdot u^{\prime}$ - вектор вихря (завихренность); $z-$ концентрация инертной примеси (т. е. примеси, не участвующей в химической реакции). По повторяющимся индексам производится суммирование, а угловые скобки означают осреднение. Подобно тому, как $\varepsilon$ характеризует уменьшение энергии турбулентности из-за вязкости, скалярная диссипация $N$ описывает, с какой скоростью происходит выравнивание неосредненных концентрационных неоднородностей из-за молекулярной диффузии. Следует отметить, что скалярная диссипация и диссипация энергии не зависят от коэффициентов молекулярного переноса и в ламинарном пограничном слое смешения между двумя плоскопараллельными потоками. Увеличение числа Рейнольдса приводит к уменьшению толщины пограничного слоя и соответствующему возрастанию градиентов скорости и концентрации. В результате (см. [20]) величины $\varepsilon$ и $N$ остаются в точности неизменными. Такая картина течения наблюдается только внутри узкого пограничного слоя (толщина слоя стремится к нулю при увеличении числа Рейнольдса), вне которого процессы молекулярного переноса несущественны, т. е. $\varepsilon=N=0$, а характеристики потока описываются уравнениями Эйлера (в ряде случаев можно использовать предположение о потенциальности течения). Описанная структура потока характерна для многих задач гидродинамики, в том смысле, что при

\footnotetext{
${ }^{3}$ Вставка в квадратных скобках авторская.
} 
больших числах Рейнольдса силы вязкости существенны лишь в очень узких областях, вне которых процессы молекулярного переноса не играют роли, что определяет перемежаемость областей, где силы вязкости существенны и где процессы молекулярного переноса не играет роли.

Аналогичная структура перемежаемости наблюдается и в турбулентном потоке. В этом случае процессы диссипации также происходят лишь в узких областях. Особенности турбулентного течения проявляются в том, что эти области хаотически перемещаются в пространстве, а значит $\langle\varepsilon\rangle$ и $\langle N\rangle$, вообще говоря, зависят от числа Рейнольдса. Согласно теории Колмогорова-Обухова [16], пространственный масштаб наиболее мелкомасштабных движений, так называемый колмогоровский, или внутренний, масштаб турбулентности, $\eta=\nu^{3 / 4}\langle\varepsilon\rangle^{-1 / 4}$. Описанное явление впервые обнаружено Корсиным [21, 22] и обычно называется перемежаемостью (полосчатостью). В настоящее время установлено, что оно характерно для всех турбулентных течений. Экспериментальные данные и результаты их теоретического анализа указывают на то, что количественное определение характеристик перемежсаемости связано с рядом приниипиальных трудностей.

\section{2. О возможности расширения подхода Кана и Хилларда на решение ря-} да задач газовой динамики. Эта статья - вторая из триптиха «кристаллизация [23], турбулентность и горение». Притом первые два фактора нас интересую в той специфике, которая связана с реконструкцией начальной стадии турбулентного диффузионного горения [21]. При реконструкции начальной стадии кристаллизации бинарных сплавов за базу [23], описывающую процесс на макроуровне, мы взяли классическую модель механики сплошных сред-модель Био насыщенной пористой среды и для описания производства флуктуаций плотности использовали механизм диффузионного расслоения $[5,9,10]$, что соответствует экспериментальным свойствам кристаллизации [8].

Для описания визуализации в гиперзвуковом потоке газа начальной стадии образования флуктуации плотности концентрации $c$ с разными скоростями, соответственно скорости газа и флуктуаций плотности (вихрей), мы используем три уравнения макроуровня: уравнения двухскоростной системы Эйлера - уравнение неразрывности для суммарной плотности и средней скорости и уравнения для импульсов газа и вихрей. Стандартно введя механизмом, действующим в зоне ударной волны, вязкость, получим уравнения

$$
\begin{aligned}
& \partial_{t} \varrho+\partial_{x}(\varrho U)=\varepsilon \partial_{x}^{2} \varrho, \\
& \partial_{t}\left((1-c) \varrho u_{1}\right)+\partial_{x}\left((1-c) \varrho u_{1}^{2}+P(c, \varrho)\right)=\varepsilon \partial_{x}^{2} u_{1}, \\
& \partial_{t}\left(c \varrho u_{2}\right)+\partial_{x}\left(c \varrho u_{2}^{2}+P(c, \varrho)\right)=\varepsilon \partial_{x}^{2} u_{2},
\end{aligned}
$$

где $\varrho_{2}=c \varrho-$ плотность флуктуаций, $\varrho_{1}=(1-c) \varrho-$ плотность инертной части газа, $P(c, \varrho)$ - давление.

Как мы отмечали выше, существующие формы моделирования турбулентности отражают две крайности - волновую (вибрационную гидродинамику) и наиболее распространенную диффузионную (статистическую) теорию турбулентности. Наша задача - построить реконструкцию начальной стадии турбулентности, промежуточную для этих двух крайностей, согласующую волновой и диффузионный характер турбулентности, и дать возможное объяс- 
нение зарождения двухскоростного режима и перемежаемости. Для реконструкции зарождения двухскоростного режима в качестве базы макроуровня (для описания волновых свойств процесса) мы используем классическую модель механики сплошных сред - регуляризацию вязкостью одной из хорошо известных форм системы Эйлера (1) для смеси [24,25], когда задана концентрация примеси.

Для описания производства флуктуаций плотности, так же как в [23], использовали механизм диффузионного расслоения - кинетическое уравнение для флуктуаций (для описания диффузионных свойств процесса)

$$
\partial_{t}(c \varrho)+\partial_{x}\left(c \varrho u_{2}\right)=\varrho K(c, T) .
$$

Здесь $K(c, T)$ - производство флуктуаций, $T$ - температура.

Мы применим расширение $[23,26,27]$ подхода Кана и Хилларда $[9,10]$ на решение задач газовой динамики. Четвертое уравнение реконструкции кинетическое уравнение мезоуровня для флуктуаций плотности (2). Явление диффузионного расслоения в зоне ударной волны моделируется при помощи производства флуктуаций плотности в уравнении (2) в форме «обобщенного химического потенциала» (уравнения Кана-Хилларда $[9,10])$

$$
K(c, T)=\partial_{x}\left(\frac{D}{T} \partial_{x} \mu\right) .
$$

Здесь $D$ - коэффициент макродиффузии, а обобщенный химический потенциал принимается в виде

$$
\mu=\Phi_{c}^{\prime}(c, T)-\varepsilon^{2} \partial_{x}^{2} c
$$

При этом коэффициент диффузии как производная химического потенциала по составу может в определенном интервале составов принимать отрицательные значения («отрицательная», или «восходящая», диффузия). Потенциал Ван-дер-Ваальса имеет вид

$$
\Phi_{c}^{\prime}(c, T)=4\left(c-c^{+}\right)\left(c-c^{c r}\right)\left(c-c^{-}\right),
$$

где $c^{ \pm}$и $c^{c r}$ - заданные параметры, зависящие от температуры, причем $c^{ \pm}$, $c^{c r} \in(0,1) ;$ симметричный потенциал имеет вид

$$
\Phi(c)=\left(c-c^{+}\right)^{2}\left(c-c^{+}\right)^{2},
$$

когда $2 c^{c r}=c^{+}+c^{-}$.

Что в этом случае позволило выдвинуть такую гипотезу механизма, действующего в зоне ударной волны? Процесс горения однородной смеси в турбулентном потоке обычно анализируется с позиции так называемой «поверхностной» модели, т. е. предполагается, что горение происходит в тонких фронтах пламени. Турбулентные пульсации, искривляя фронт пламени, увеличивают его поверхность, а движение фронта пламени по нормали со скоростью нормального распространения пламени $u_{n}$ приводит к уменьшению площади этой поверхности. Этот процесс должен, очевидно, характеризоваться двумя параметрами: пульсационной скоростью $u^{\prime}$ и величиной $u_{n}$. При одинаковых значениях $u_{n}$ и $u^{\prime}$ скорость турбулентного распространения пламени $u_{T}$ 
в «богатых» и «бедных» смесях оказывается существенно различной. В работах $[28,29]$ отмечается, что при одинаковых значениях скорости нормального распространения пламени $u_{n}$ в богатых смесях водорода и метана с воздухом величина $u_{n}$ ниже, чем в бедных. У пропано-воздушных смесей наблюдается противоположная картина. Отмеченное обстоятельство естественно связать с поведением эффективного коэффициента молекулярного переноса при изменении состава смеси. В связи с этим напомним, что при увеличении коэффициента $\alpha$ избытка воздуха эффективный коэффициент молекулярного переноса $a(\alpha)$ в первом случае уменьшается, а во втором возрастает. Такой эффект обусловлен диффузионным расслоением, т. е. неравенством коэффиииентов дифбузии окислителя и горючего.

Наличие диффузионного расслоения особенно наглядно демонстрируется в приближении сильного разбавления [30], когда в смеси в большом количестве находится инертное вещество. Это приближение с хорошей точностью пригодно при анализе процессов горения углеводородных горючих в воздухе, когда содержание азота в смеси достаточно велико (порядка 80\%). В работе [31] доказано наличие диффузионного расслоения в сферически симметричном случае.

Качественные соображения о том, что диффузионное расслоение может существенно влиять на скорость распространения пламени в развитом турбулентном потоке, содержатся в работе [32]. На первый взгляд, такие представления кажутся малоправдоподобными, так как хорошо известно, что структура развития турбулентности и процессы турбулентного переноса практически не зависят от числа Рейнольдса и Пекле. Однако следует иметь в виду, что в турбулентном потоке горение происходит в узких зонах, сильно искривленных турбулентными пульсациями. При увеличении числа Рейнольдса появляются все более мелкие вихри, которые должны все сильнее и сильнее искривлять фронт пламени и тем самым увеличивать диффузионное расслоение. Несмотря на то, что механизм этого явления в турбулентном потоке не вполне ясен, полученные значения эффективного коэффициента молекулярного переноса позволяют обобщать данные измерений скорости турбулентного распространения пламени $u_{T}$ в турбулентных потоках разных газов. В качестве примера приведем результаты обработки данных [29], которые получены при измерении $u_{T}$ в сферической бомбе. Турбулентность создавалась вращением мешалок, расположенных на стенке бомбы. Использовались смеси водорода, метана и пропана с воздухом.

Процесс горения однородной смеси в турбулентном потоке обычно анализируется с позиции так называемой «поверхностной» модели, т. е. предполагается, что горение происходит в тонких фронтах пламени. Турбулентные пульсации, искривляя фронт пламени, увеличивают его поверхность, а движение фронта пламени по нормали со скоростью нормального распространения пламени $u_{n}$ приводит к уменьшению площади этой поверхности. Этот процесс должен, очевидно, характеризоваться двумя параметрами: пульсационной скоростью $u^{\prime}$ и величиной $u_{n}$. При одинаковых значениях $u_{n}$ и $u^{\prime}$ скорость турбулентного распространения пламени $u_{T}$ в «богатых» и «бедных» смесях оказывается существенно различной. Для объяснения этого факта в работе [31] сделано предположение, что величина $u_{T}$ также должна зависеть от толщины $\delta$ ламинарного фронта пламени. Эту зависимость удобно характеризовать с помощью эффективного коэффициента молекулярного переноса 
$a$, определяемого как $\delta u_{n}$. Величина $a$ находится путем обработки опытных данных, в которых измерялись минимальная энергия искрового воспламенения, критический градиент скорости при проскоке пламени, гасящий диаметр и т. д. Расположение экспериментальных точек (для водорода, метана,пропана) вокруг единой кривой свидетельствует о том, что диффузионное расслоение при горении в турбулентном потоке можно хорошо описать с помощью введенного коэффициента молекулярного переноса.

Предложенная гипотеза механизма, действующего в зоне ударной волны, опирается на предположение, что существенную роль в формировании флуктуаций плотности играют диффузионное расслоение и возникновение двухскоростного режима (катастрофа Римана-Гюгонио). Реконструкция этих базовых свойств потока, диффузионной и волновой характеристик потока и их согласование станут предметом наших исследований. Как мы покажем ниже, возникновение двухскоростного режима (катастрофа Римана-Гюгонио) связано с потерей строгой гиперболичности многоскоростной системы Эйлера (волновая природа процесса), в то время как эффект рождения перемежаемости связан с предположением о существенной роли в формировании флуктуаций плотности диффузионного расслоения (диффузионная природа процесса). Последнее вводится кинетическим уравнением производства флуктуаций плотности. Потенциал $\Phi$ определяется спинодалями $c^{ \pm}(T)$, существование которых следует из утверждения Пригожина, что возникновение двухскоростного потока можно интерпретировать как «переходы при множественных стационарных состояниях по закону "все или ничего" и теория катастроф» и определяется экспериментом.

Суммарно (1), (2) дают нестандартную регуляризацию [6] классической системы уравнений Эйлера [24,25]:

$$
\begin{aligned}
& \partial_{t} \varrho+\partial_{x}(\varrho U)=0, \\
& \partial_{t}\left((1-c) \varrho u_{1}\right)+\partial_{x}\left((1-c) \varrho u_{1}^{2}+P(c, \varrho)\right)=0, \\
& \partial_{t}\left(c \varrho u_{2}\right)+\partial_{x}\left(c \varrho u_{2}^{2}+P(c, \varrho)\right)=0, \\
& \partial_{t}(c \varrho)+\partial_{x}\left(c \varrho u_{2}\right)=0 .
\end{aligned}
$$

В чем нестандартность регуляризации (1), (2)? Для этих целей мы прежде всего в системе (3) перейдем к новым переменным $c, \varrho, U, u_{2}$, в которых система (3) примет следующий вид:

$$
\begin{aligned}
& \partial_{t} c+U \partial_{x} c-\partial_{x}\left(c\left(U-u_{2}\right)\right)-c\left(U-u_{2}\right) \varrho^{-1} \partial_{x} \varrho=0, \\
& \partial_{t} \varrho+\partial_{x}(\varrho U)=0, \\
& \partial_{t}(\varrho U)+\partial_{x}\left(\varrho U^{2}+2 P(c, \varrho)\right)+\partial_{x}\left(\frac{c}{1-c} \varrho\left(U-u_{2}\right)^{2}\right)=0, \\
& \partial_{t}\left(c \varrho u_{2}\right)+\partial_{x}\left(c \varrho u_{2}^{2}+P(c, \varrho)\right)=0 .
\end{aligned}
$$

Систему (4) будем называть приведенной системой Эйлера. Ее регуляризация 
(1), (2) запишется в виде

$$
\begin{aligned}
& \partial_{t} c+U \partial_{x} c-\partial_{x}\left(c\left(U-u_{2}\right)\right)-c\left(U-u_{2}\right) \varrho^{-1} \partial_{x} \varrho=K(c, T), \\
& \partial_{t} \varrho+\partial_{x}(\varrho U)=\varepsilon \partial_{x}^{2} \varrho, \\
& \partial_{t}(\varrho U)+\partial_{x}\left(\varrho U^{2}+2 P(c, \varrho)\right)+\partial_{x}\left(\frac{c}{1-c} \varrho\left(U-u_{2}\right)^{2}\right)=\varepsilon \partial_{x}^{2} U, \\
& \partial_{t}\left(c \varrho u_{2}\right)+\partial_{x}\left(c \varrho u_{2}^{2}+P(c, \varrho)\right)=\varepsilon \partial_{x}^{2} u_{2} .
\end{aligned}
$$

В дальнейшем для простоты мы рассмотрим изотермический случай, когда $T=$ const, и случай, когда температура совершенного газа $T=P / \kappa \varrho$.

Что в такой постановке общего с классикой, а что нет?

1) нестандартность определения слабого решения для приведенной системы Эйлера связана с тем, что первое уравнение умножается на тестовую функцию $c(x, t) \varphi(x, t)$, где $\varphi(x, t) \in C_{0}^{\infty}\left(\mathbb{R}^{2}\right)$ - бесконечно дифференцируемая функция в $\mathbb{R}^{2}$ с компактным носителем, в то время как три уравнения для усредненной плотности, $U$ и $u_{2}$ стандартно умножаются на тестовые функции $\psi_{j}(x, t) \in C_{0}^{\infty}\left(\mathbb{R}^{2}\right), j=1,2,3$, соответственно;

2) есть четыре семейства волн разрежения и четыре семейства устойчивых ударных волн (см. [6]), три семейства устойчивых ударных волн (5), есть поднятие устойчивых ударных волн усеченной системы Әйлера.

Система (1), (2) является регуляризацией в смысле приведенного выше определения слабого решения, поскольку правая часть - интегралы

$$
\begin{gathered}
\int_{\mathbb{R}_{+}^{2}} \varepsilon \partial_{x} \varrho \partial_{x} \psi_{1}(x, t) d x d t \rightarrow 0, \quad \int_{\mathbb{R}_{+}^{2}} \varepsilon \partial_{x} U \partial_{x} \psi_{2}(x, t) d x d t \rightarrow 0, \\
\int_{\mathbb{R}_{+}^{2}} \varepsilon \partial_{x} u_{2} \partial_{x} \psi_{3}(x, t) d x d t \rightarrow 0, \quad \int_{\mathbb{R}_{+}^{2}} \frac{D}{T} \partial_{x} \mu \partial_{x}(c(x, t) \varphi(x, t)) d x d t \rightarrow 0
\end{gathered}
$$

при $\varepsilon \rightarrow 0$.

На основе приведенной выше нестандартной регуляризации системы (4) уравнений Эйлера с использованием вязкости и введения отрицательной диффузии мы приведем визуализацию самовозбуждающихся режимов для сверхзвукового потока с достаточно большим числом Маха. Хорошо известно, что система уравнений Эйлера имеет много решений. А нет ли среди них решений, естественно отличных от вязких, которые обладали бы свойствами дальнодействия и самовозбуждения, и как их выделить?

3. Катастрофа Римана-Гюгонио. Начнем с пункта 1) двухскоростное течение (катастрофа Римана-Гюгонио), реконструкции базовых свойств начальной стадии турболенизации.

Что общего в задаче Римана для приведенной системой Эйлера с классикой, а что нет? Есть четыре семейства волн разрежения и четыре семейства устойчивых ударных волн (см. [6,7]). Три семейства устойчивых ударных волн (4) - поднятие устойчивых ударных волн усеченной системы Эйлера:

$$
\begin{aligned}
& \partial_{t} \varrho+\partial_{x}(\varrho U)=0, \\
& \partial_{t}(\varrho U)+\partial_{x}\left(\varrho U^{2}+2 P(c, \varrho)\right)+\partial_{x}\left(\frac{c}{1-c} \varrho\left(U-u_{2}\right)^{2}\right)=0, \\
& \partial_{t}\left(c \varrho u_{2}\right)+\partial_{x}\left(c \varrho u_{2}^{2}+P(c, \varrho)\right)=0 .
\end{aligned}
$$


Чем интересна усеченная система Эйлера (6)? Система (6) дает пример нестрого гиперболической системы законов сохранения, для которых на критическом многообразии (кратных собственных значений) нет полного базиса собственных векторов, но есть присоединенный вектор. Построенные ниже бифуркации критических ударных волн дают ответ на давно поставленную Лаксом задачу о том, что происходит с ударной волной при прохождении ею в фазовом пространстве критического многообразия? Процесс остается волновым. В фазовой плоскости переменных $\left(\varrho, U, u_{2}\right)$ выделяется множество точек, не достижимых цепочками устойчивых ударных волн и волн разрежения. Вход в это множество приводит к появлению бифуркации фронта ударной волны компоненты $u_{2}$. При любом фиксированном $c \in(0,1)$ при специальном подборе начальных данных [6,7] можно получить неклассическое решение задачи Римана с бифуркацией фронта ударной волны компоненты в два фронта, когда для переднего фронта выполнено условие Гюгонио, в то время как для заднего фронта уравнение для скорости имеет другой характер. Численно такая бифуркация однофронтового решения для упрощенной усеченной системы

$$
\begin{aligned}
& \partial_{t} \varrho+\partial_{x}(\varrho U)=0, \\
& \partial_{t}(\varrho U)+\partial_{x}\left(\varrho U^{2}+2 P(c, \varrho)\right)=0, \\
& \partial_{t}\left(c \varrho u_{2}\right)+\partial_{x}\left(c \varrho u_{2}^{2}+P(c, \varrho)\right)=0
\end{aligned}
$$

без члена $\partial_{x}\left(\frac{c}{1-c} \varrho\left(U-u_{2}\right)^{2}\right)$ во втором уравнении (6) получена в [6]. Ниже мы приводим результаты численного моделирования, приведенного в [33]. На рис. $1, b$ - укрупнение динамики бифуркации фронта устойчивой ударной волны скорости второй компоненты $u_{2}$.

Как показало численное моделирование [33], в фазовой плоскости переменных выделяется множество точек, не достижимых цепочками устойчивых ударных волн и волн разрежения. Вход в это множество приводит к появлению бифуркации фронта ударной волны компоненты $u_{2}$. При любом фиксированном наборе специально подобранных начальных данных можно получить неклассическое решение задачи Римана (так называемый горбатый кинк), которое представляет катастрофу Римана-Гюгонио. Полученные бифуркации типа многогорбых кинков имеют сверхзвуковой характер, связанный с критической скоростью $\omega^{c r}=\sqrt{4[P] / c[\varrho]}$, большей скорости звука. В общем случае для полной усеченной системы (6) бифуркации фронта ударной волны усредненной скорости $U$ и усредненной плотности $\varrho$ имеют тот же профиль. 4

4. Согласование неустойчивостей. Теперь перейдем к реконструкции пункта 4) базовых свойств начальной стадии турбулентности: согласование катастрофы Римана-Гюгонио и неустойчивостей, порождающих образование флуктуаций плотности. При исследовании усеченной модели Эйлера мы получили семейства бифуркаций верхнего и нижнего критический решений, определяемые, при фиксированном значении $\omega$, выбором либо значения второй скорости $\omega_{1} \in(0, \omega)$, либо начальной амплитуды ударной волны флуктуаций - значения $a_{0}^{+}$(см. $\left.[6,23]\right)$. Эта многозначность связана с тем, что мы не описали неустойчивость, рождающую всплеск $\left(a_{0}^{+}\right)$в стадии зарож-

\footnotetext{
${ }^{4}$ Этот результат будет опубликован в ближайшей работе.
} 

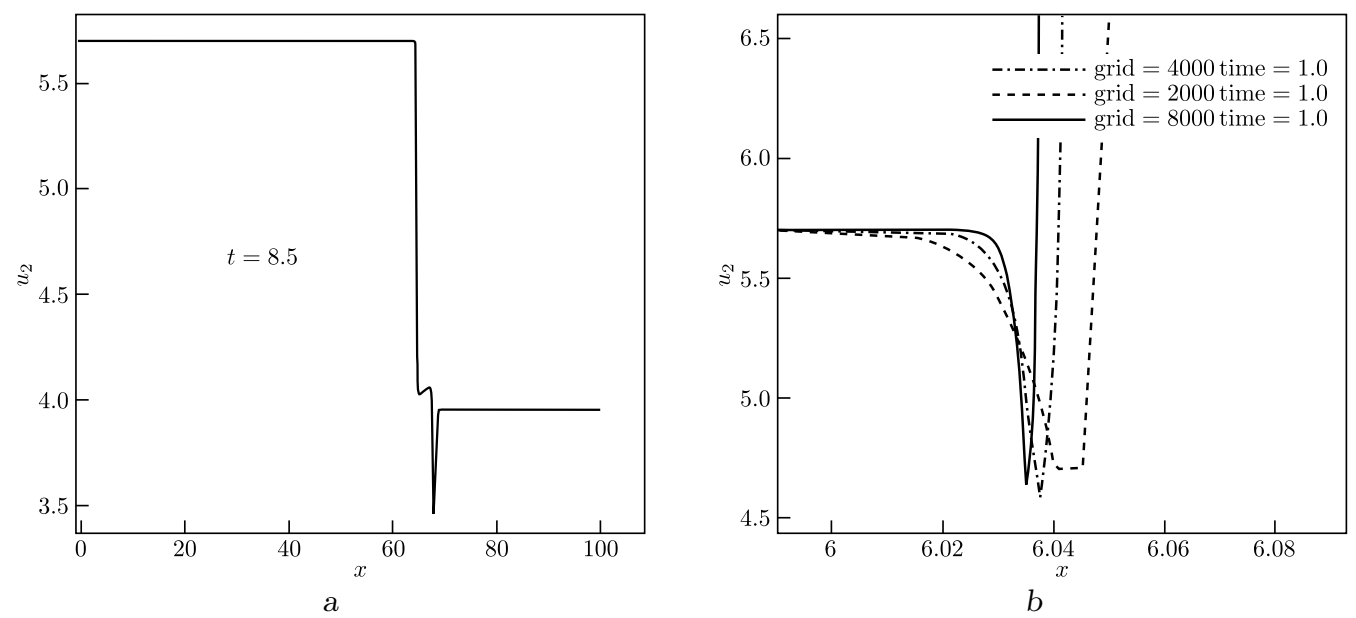

Рис. 1. [Figure 1]
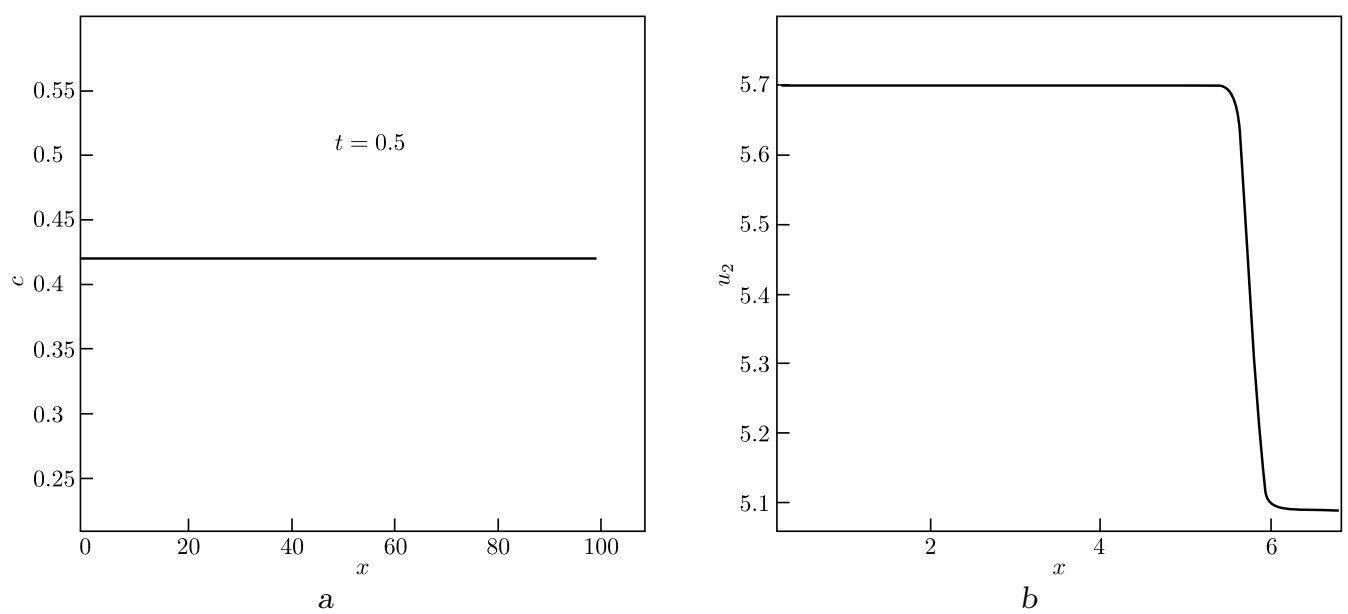

Рис. 2. [Figure 2]

дения бифуркации для усеченной системы Әйлера. Из численного моделирования (см. [33]) следует, что в полной приведенной системе это можно отнести на счет неустойчивости константы $c_{0}$ в данных Коши концентрации $c$ активной компоненты из области лабильности уравнения Кана-Хилларда (см. рис. 2, а).

Начальный всплеск (рис. 3, а) определяется уравнением для концентрации в приведенной системе и согласуется с всплеском бифуркации устойчивой однофронтовой волны $u_{2}$ (рис. $\left.3, b\right)$.

Возникающий промежуток между передним и задним фронтами ударной волны для $u_{2}$ заполняется осцилляциями самовозбуждающегося решения (рис. $4, b)$. В общем случае для полной усеченной системы (6) бифуркации фронта ударной волны усредненной скорости $U$ и усредненной плотности $\varrho$ имеют тот же профиль.

5. Перемежаемость. Как видим, диссипация энергии $\varepsilon$ проходит в зоне бифуркации, между фронтами флуктуации скорости $u_{2}$. Процессы диссипации происходят лишь в узких областях, что соответствует определению переме- 

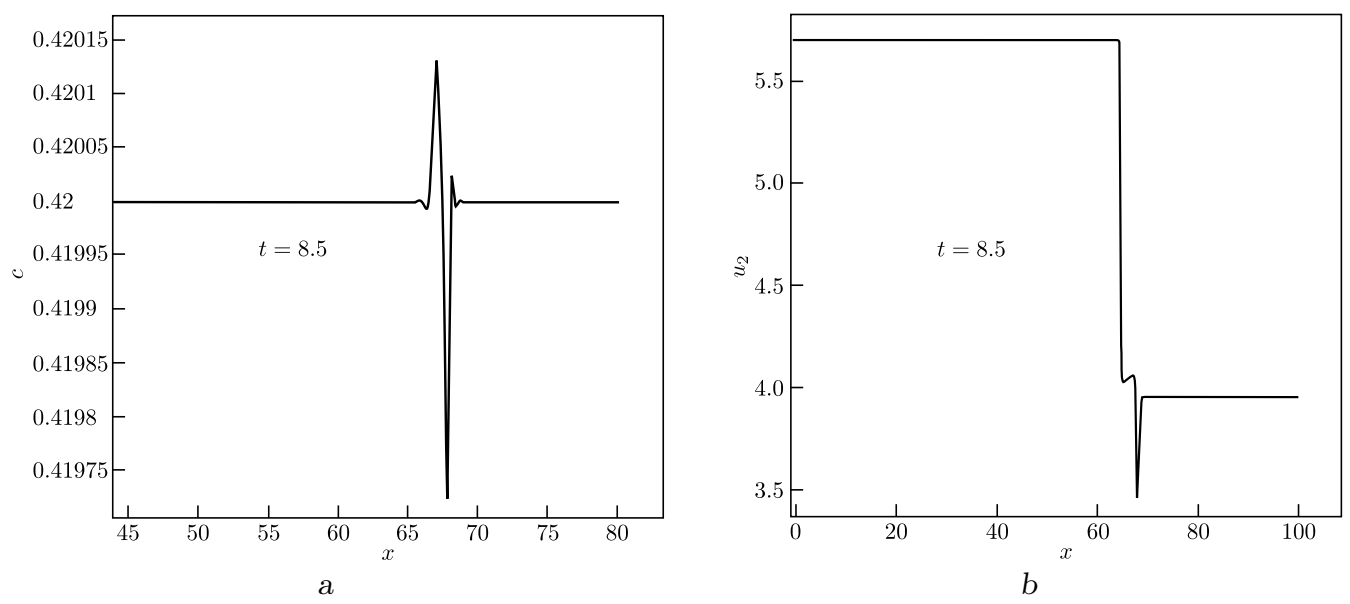

Рис. 3. [Figure 3]
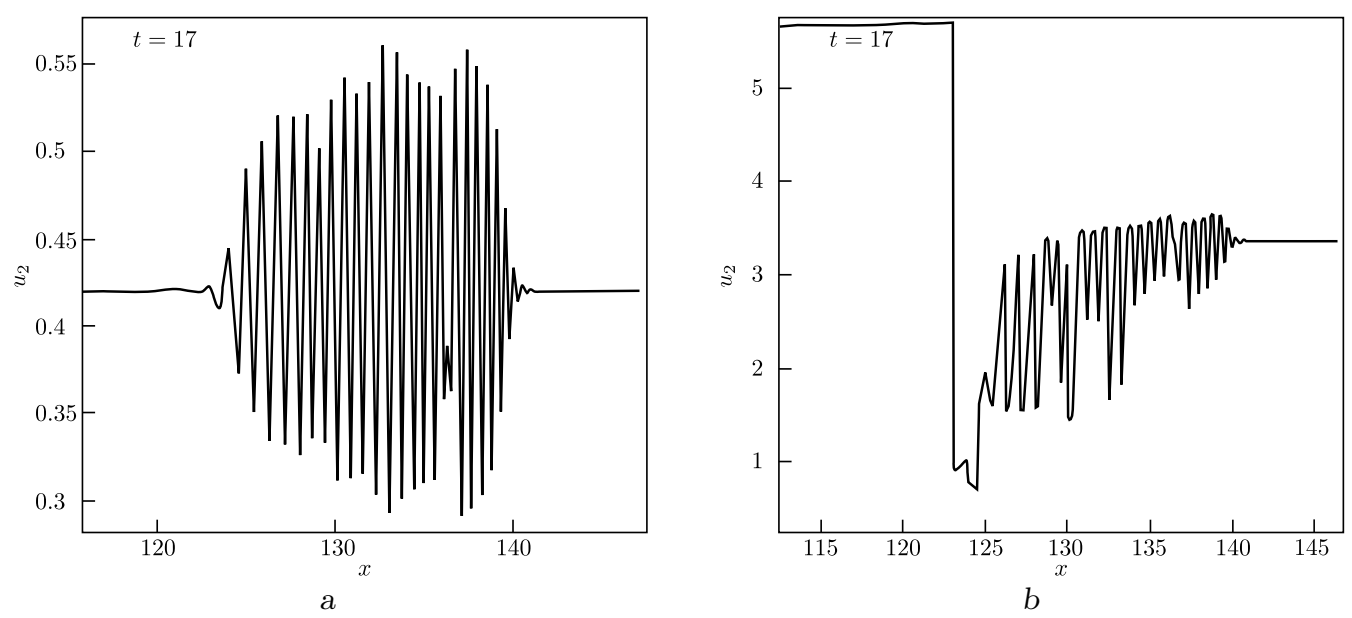

Рис. 4. [Figure 4]

жаемости (полосчатости) течения. Этот факт подтверждает утверждение 4) списка базовых свойств внутренней турбулентности.

В силу приведенного выше определения слабого решения предельный переход при $[c] \rightarrow 0$ трех семейств ударных волн, определяемых устойчивыми ударными волнами усеченной системы Эйлера, дает три семейства устойчивых ударных волн, для которых первое приближение $c=c_{0}=$ const и $\left(\varrho, U, u_{2}\right)$ - решение усеченной системы Эйлера. Регуляризация (5) для многокомпонентной системы уравнений Эйлера позволяет выделить в ней невязкие решения, описывающие процессы с избыточной энергией (в мезомасштабе), которые прежде всего характеризуются дальнодействием и самовозбуждающимися режимами (например, коагуляция, начальная стадия кристаллизации сплавов). Нестандартные регуляризации классических моделей механики сплошных сред встречались и ранее (см. [13]).

6. О природе бифуркаций односкоростных (однофронтовых) решений задачи Римана усеченной системы Эйлера. Как мы отмечали выше, основу «странного» поведения решений регуляризации (5) составляют бифуркации 
односкоростных (однофронтовых) решений усеченной системы Эйлера в двухскоростные (двухфронтовые) решения. В статье [7] для упрощенной усеченной системы Эйлера (6) приведены результаты, аналитически доказывающие существование бифуркаций односкоростных решений в двухскоростные решения. Перейдем к исследованию условий существования двухфронтовых решений усеченной системы Эйлера. Рассмотрим упрощенную усеченную систему

$$
\begin{aligned}
& \partial_{t} \varrho+\partial_{x}(\varrho U)=0, \\
& \partial_{t}(\varrho U)+\partial_{x}\left(\varrho U^{2}+2 P(c, \varrho)\right)=0, \\
& \partial_{t}\left(c \varrho u_{2}\right)+\partial_{x}\left(c \varrho u_{2}^{2}+P(c, \varrho)\right)=0
\end{aligned}
$$

и задачу Римана для $V=\left(\varrho, U, u_{2}\right)^{\mathrm{T}}$ системы $(7)$, т.е. задачу Коши с начальными условиями

$$
\left.V\right|_{t=0}= \begin{cases}V_{-}, & x<0 \\ V_{+}, & x>0\end{cases}
$$

Ее регуляризация вязкостью имеет вид

$$
\begin{aligned}
& \partial_{t} \varrho+\partial_{x}(\varrho U)=\varepsilon \partial_{x}^{2} \varrho, \\
& \partial_{t}(\varrho U)+\partial_{x}\left(\varrho U^{2}+2 P(c, \varrho)\right)=\varepsilon \partial_{x}^{2} U, \\
& \partial_{t}\left(c \varrho u_{2}\right)+\partial_{x}\left(c \varrho u_{2}^{2}+P(c, \varrho)\right)=\varepsilon \partial_{x}^{2} u_{2} .
\end{aligned}
$$

Предположим, что выполнены следующие условия знаков:

$$
[\varrho]<0, \quad[U]<0, \quad\left[u_{2}\right]<0
$$

а также имеет место уравнение состояния

$$
P(\varrho)=P_{0} \varrho^{\gamma}
$$

где $P_{0}>0, \gamma>1$ - заданные константы. Кроме того, будем предполагать, что имеет место неравенство

$$
0<\omega_{1}<\omega
$$

6.1. Алгебраическое исследование. Перепишем систему (7) в форме Коши:

$$
\begin{aligned}
& \partial_{t} \varrho+U \partial_{x} \varrho+\varrho \partial_{x} U=0, \\
& \partial_{t} U+\frac{2 P^{\prime}}{\varrho} \partial_{x} \varrho+U \partial_{x} U=0, \\
& \partial_{t} u_{2}+\frac{c_{0} u_{2}\left(u_{2}-U\right)+P^{\prime}}{c_{0} \varrho} \partial_{x} \varrho-u_{2} \partial_{x} U+2 u_{2} \partial_{x} u_{2}=0
\end{aligned}
$$

или в матричном виде:

$$
\partial_{t} V+\mathscr{A} \partial_{x} V=\varepsilon \partial_{x}^{2} V
$$

где

$$
\mathscr{A}=\left(\begin{array}{ccc}
U & \varrho & 0 \\
2 P^{\prime} / \varrho & U & 0 \\
\left(c_{0} u_{2}\left(u_{2}-U\right)+P^{\prime}\right) /\left(c_{0} \varrho\right) & -u_{2} & 2 u_{2}
\end{array}\right) .
$$


Собственные значения этой матрицы следующие:

$$
\lambda_{ \pm}=U \pm \sqrt{2 P^{\prime}}, \quad \lambda_{3}=2 u_{2}
$$

При $\varrho>0$ происходит нарушение строгой гиперболичности на гиперповерхностях

$$
\Sigma_{ \pm}=\left\{\left(\varrho, U, u_{2}\right) \mid 2 u_{2}=U \pm \sqrt{2 P^{\prime}}\right\}
$$

Рассмотрим окрестность гиперповерхности $\Sigma_{+}$(для $\Sigma_{-}$все аналогично). Обозначим

$$
\mathscr{A}_{ \pm}=\mathscr{A}-\lambda_{ \pm} E \text {. }
$$

Тогда на критической поверхности $\Sigma_{+}$имеем

$$
\mathscr{A}_{ \pm} \mid \Sigma_{+}=\left(\begin{array}{ccc}
\mp \sqrt{2 P^{\prime}} & \varrho & 0 \\
2 P^{\prime} / \varrho & \mp \sqrt{2 P^{\prime}} & 0 \\
\left(c_{0} u_{2}\left(\sqrt{2 P^{\prime}}-u_{2}\right)+P^{\prime}\right) /\left(c_{0} \varrho\right) & -u_{2} & \sqrt{2 P^{\prime}} \mp \sqrt{2 P^{\prime}}
\end{array}\right) .
$$

Если для матрицы $\left.\mathscr{A}_{+}\right|_{\Sigma_{+}}$выполнено условие жордановости

$$
c_{0} u_{2}^{2}-P^{\prime} \neq 0
$$

то ранг матрицы $\mathscr{A}_{+} \mid \Sigma_{+}$равен двум, т.е. значению $\lambda_{+}$соответствует жорданова клетка размера 2. Соответствующий собственный вектор $\nu=(0,0,1)^{\mathrm{T}}$ будем называть критическим. Для матрицы $\mathscr{A}_{-}$собственный вектор, соответствующий нулевому собственному значению, имеет вид

$$
\nu_{-}=\left(\varrho,-\sqrt{2 P^{\prime}}, \alpha_{-}\right)^{\mathrm{T}} \text {, }
$$

где

$$
\alpha_{-}=-u_{2}+\frac{u_{2}^{2}}{2 \sqrt{2 P^{\prime}}}-\frac{\sqrt{2 P^{\prime}}}{4 c_{0}} .
$$

Нетрудно показать (см. [6]), что проекция множества нарушения строгой гиперболичности $\Sigma_{+}$на плоскость переменных $\left(U, u_{2}\right)$ разделяет плоскость переменных $\left(U, u_{2}\right)$ на две открытых части. Отсюда следует несправедливость теоремы А. Майда [34] для усеченной системы Эйлера (7).

6.2. Устойчивые ударные волны. Решения системы (8) будем искать в виде бегущих волн. Для случая, когда решение имеет один общий фронт, положим

$$
\varrho=\varrho\left(\frac{x-x^{*}(t)}{\varepsilon}\right), \quad U=U\left(\frac{x-x^{*}(t)}{\varepsilon}\right), \quad u_{2}=u_{2}\left(\frac{x-x^{*}(t)}{\varepsilon}\right) .
$$

Для случая двухфронтового решения положим

$$
\begin{gathered}
\varrho=\varrho\left(\frac{x-x^{*}(t)}{\varepsilon}\right), \quad U=U\left(\frac{x-x^{*}(t)}{\varepsilon}\right), \\
u_{2}=a\left(\frac{x-x^{*}(t)}{\varepsilon}\right)+b\left(\frac{x-x_{1}(t)}{\varepsilon}\right) .
\end{gathered}
$$


Обозначим также

$$
\dot{x}^{*}(t)=\omega, \quad \dot{x}_{1}(t)=\omega_{1} .
$$

Потребуем, чтобы для однофронтовых решений имело место условие стабилизации:

$$
\begin{aligned}
& \varrho( \pm \infty)=\varrho_{ \pm}, \quad U( \pm \infty)=U_{ \pm}, \quad u_{2}( \pm \infty)=u_{2, \pm}, \\
& \dot{\varrho}( \pm \infty)=0, \quad \dot{U}( \pm \infty)=0, \quad \dot{u}_{2}( \pm \infty)=0 .
\end{aligned}
$$

Для двухфронтовых решений условие стабилизации имеет вид

$$
\begin{array}{lll}
\varrho( \pm \infty)=\varrho_{ \pm}, & U( \pm \infty)=U_{ \pm}, & a(-\infty)=u_{2,-}, \\
b(-\infty)=0, & (a+b)(+\infty)=u_{2,+} .
\end{array}
$$

После подстановки вида однофронтового решения (11) в (8) получаем систему ОДУ

$$
\begin{aligned}
& -\omega\left(\varrho-\varrho_{-}\right)+\left(\varrho U-\varrho_{-} U_{-}\right)=\dot{\varrho}, \\
& -\omega\left(\varrho U-\varrho_{-} U_{-}\right)+\left(\varrho U^{2}-\varrho_{-} U_{-}^{2}+2\left(P-P_{-}\right)\right)=\dot{U}, \\
& { }_{0} \omega\left(\varrho u_{2}-\varrho_{-} u_{2,-}\right)+c_{0}\left(\varrho u_{2}^{2}-\varrho_{-} u_{2,-}^{2}+P-P_{-}\right)=\dot{u}_{2} .
\end{aligned}
$$

Проинтегрировав эту систему и воспользовавшись условиями стабилизации (11), получаем условия Рэнкина-Гюгонио для однофронтового случая:

$$
\begin{aligned}
& -\omega[\varrho]+[\varrho U]=0, \\
& -\omega[\varrho U]+\left[\varrho U^{2}+2 P\right]=0, \\
& -\omega\left[c_{0} \varrho u_{2}\right]+\left[c_{0} \varrho u_{2}^{2}+P\right]=0 .
\end{aligned}
$$

Заметим, что регуляризованная система (8) допускает факторизацию: первые два уравнения этой системы не содержат неизвестной функции $u_{2}$. После подстановки явного вида двухфронтового решения соответствующая первым двум уравнениям (8) система ОДУ имеет вид

$$
\begin{aligned}
& -\omega \dot{\varrho}+(\varrho U)=\ddot{\varrho} \\
& -\omega\left(\varrho U \dot{)}+\left(\varrho U^{2}+2 P \dot{)}=\ddot{U} .\right.\right.
\end{aligned}
$$

Рассмотрим теперь ОДУ, соответствующее третьему уравнению системы (8). После подстановки вида двухфронтового решения (10) получаем

$$
-\omega c_{0}(\varrho(a+b)+\varrho \dot{a})-\omega_{1} c_{0} \varrho \dot{b}+\left(c_{0} \varrho(a+b)^{2}+\dot{P}\right)=(a+\ddot{b}) .
$$

После перегруппировки это уравнение принимает вид

$$
-\omega c_{0}(\varrho \dot{a})-\omega_{1} c_{0}(\varrho \dot{b})+\left(\omega_{1}-\omega\right) c_{0} \varrho \dot{b} b+\left(c_{0} \varrho(a+b)^{2}+\dot{P}\right)=(a+\ddot{b}) .
$$

Аналогично тому, как было получено условие Рэнкина-Гюгонио для однофронтового решения, получим

$$
\begin{aligned}
& -\omega[\varrho]+[\varrho U]=0, \\
& -\omega[\varrho U]+\left[\varrho U^{2}+2 P\right]=0, \\
& -\omega\left[\varrho u_{2}\right]+\left(\omega-\omega_{1}\right)[b] \varrho-+\left[\varrho u_{2}^{2}+P / c_{0}\right]=0 .
\end{aligned}
$$


При этом в последнем уравнении мы воспользовались равенством

$$
\int_{-\infty}^{+\infty} \dot{\varrho}(\tau) b(\tau) d \tau=[b] \varrho_{-},
$$

которое имеет место при условии (9).

6.3. Бифуркации критических однофронтовых решений. В этом разделе мы приведем построение бифуркаций однофронтового решения в двухфронтовое как возмущение критических однофронтовых решений усеченной системы. Бифуркация реализуется распадом решения на две бегущие волны. Каков сценарий бифуркации? Задача этого параграфа - найти условия бифуркации однофронтового решения в двухфронтовое, т. е. природу разветвления фронта ударной волны. Мы покажем ниже, что бифуркация возникает как возмущение критических решений $\left(\varrho^{c r}, U^{c r}, u_{2}^{c r}\right)$, исследованных выше. Система (8) факторизуется, поэтому исследуем сначала первые два уравнения:

$$
\begin{aligned}
& -\omega\left(\varrho-\varrho_{-}\right)+\varrho U-\varrho_{-} U_{-}=\dot{\varrho} \\
& -\omega\left(\varrho U-\varrho_{-} U_{-}\right)+\varrho U^{2}-\varrho_{-} U_{-}^{2}+2\left(P-P_{-}\right)=\dot{U} .
\end{aligned}
$$

Определим условия Лакса, при которых есть сепаратрисное решение, т. е. существует пара монотонных функций $(\varrho, U)$, являющаяся решением системы ОДУ (13) и удовлетворяющая условиям стабилизации

$$
\begin{aligned}
& \varrho( \pm \infty ; t)=\varrho_{ \pm}(t), \quad U( \pm \infty ; t)=U_{ \pm}(t), \\
& \stackrel{\circ}{\varrho}( \pm \infty ; t)=0, \quad \stackrel{\circ}{U}( \pm \infty ; t)=0
\end{aligned}
$$

с условиями Гюгонио

$$
\begin{aligned}
& -\omega[\varrho]+[\varrho U]=0, \\
& -\omega[\varrho U]+\left[\varrho U^{2}\right]=0
\end{aligned}
$$

где $\omega=\dot{x}^{*}$. Из условий Гюгонио получаем

$$
\omega=\frac{[\varrho U]}{[\varrho]}=U_{-}+\varrho_{+} \frac{[U]}{[\varrho]} .
$$

В дальнейшем рассматриваем случай, когда $[\varrho]<0,[U]<0$. Как мы установили выше, собственные значения усеченной системы

$$
\lambda_{1}(\tau)=U-\sqrt{P^{\prime}}, \quad \lambda_{2}(\tau)=U+\sqrt{P^{\prime}}, \quad \lambda_{3}(\tau)=2 u_{2}
$$

Положим $\lambda_{j}^{ \pm}=\lambda_{j}( \pm \infty)$. Теперь сформулируем условие Лакса существования сепаратрисного решения системы (усредненных компонент).

6.4. Условие устойчивости Лакса (см. [35].) Пусть для $\omega$ выполнено неравенство для ударной волны первого семейства

$$
\lambda_{1}^{+}<\lambda_{2}^{+}<\omega \quad \lambda_{1}^{-}<\omega<\lambda_{2}^{-},
$$


которое определяет интервал устойчивых скоростей $\omega$ для ОДУ усредненных параметров $(\varrho, U)$. Условие $(14)$ гарантирует существование стабилизирующегося решения системы ОДУ (13) для усредненных параметров. Приведем условия, когда эти неравенства справедливы для скорости $\omega$, определяемой условием Гюгонио. Если выполнены условия

$$
\varrho>0, \quad U>0, \quad[\varrho]<0, \quad[U]<0
$$

и дополнительно справедливо неравенство

$$
\varrho_{-}-2 \varrho_{+}<0
$$

то скорость $\omega$, определяемая условиями Гюгонио, удовлетворяет неравенствам (14) условия Лакса для подсистемы первых двух уравнений (12) усредненных компонент. В дальнейшем будем считать $\omega$ выбранным условие Гюгонио однофронтовой задачи.

6.5. Нарушение условия Лакса. Теперь докажем существование двух критических решений системы ОДУ (8). Пусть в фазовом пространстве ось $u_{2}$ направлена вверх, перпендикулярно плоскости усредненных переменных $(\varrho, U)$. Пусть для $\omega$ выполнены неравенства для ударной волны первого семейства

$$
\lambda_{1}^{+}<\lambda_{2}^{+}<\omega<\lambda_{3}^{+}, \quad \lambda_{1}^{-}<\omega<\lambda_{2}^{-}<\lambda_{3}^{-},
$$

которые гарантируют существование стабилизирующегося решения системы ОДУ (8). Здесь возможны два сценария управления устойчивыми ударными волнами (и их регуляризациями) для получения критических решений системы (8). Одно критическое решение, которое назовем верхним, получим управлением левым предельным значением $\left(\varrho_{-}, U_{-}, u_{2,-}\right)^{\perp}$, находясь выше верхней ветви критического многообразия $\Sigma_{+}$, уменьшая $u_{2,-}$ до первого выхода на критическое многообразие $\Sigma^{+}$в точке $\left(\varrho_{+}^{c r}, U_{+}^{c r}, u_{2,+}^{c r}\right)$. Второе критическое решение, которое назовем нижним, получим, если в фазовой плоскости будем управлять левым предельным значением $\left(\varrho_{-}, U_{-}, u_{2,-}\right)^{\perp}$, находясь ниже верхней ветви критического многообразия $\Sigma_{+}$, и увеличивая $u_{2,-}$ до первого выхода на критическое многообразие $\Sigma^{+}$в точке $\left(\varrho_{-}^{c r}, U_{-}^{c r}, u_{2,-}^{c r}\right)$.

Теперь отметим, что при выполнении условий (15), (16) и

$$
\omega^{2}-\frac{4[P]}{c_{0}[\varrho]}>0,
$$

для критического значения управления

$$
u_{2,-}^{c r}=\frac{1}{2}\left(\omega+\sqrt{\frac{|[\varrho]|}{\varrho-}\left(\omega^{2}-\frac{4[P]}{c_{0}[\varrho]}\right)}\right)
$$

существует монотонно убывающее стабилизирующееся решение системы (8), для которого $\left(\varrho_{+}^{c r}, U_{+}^{c r}, u_{2,+}^{c r}\right)$ принадлежат верхней ветви критического многообразия $\Sigma_{+}$, и $u_{2,+}^{c r}=\omega / 2$. Более того, не существует однофронтовое монотонно убывающее стабилизирующееся решения системы (8) с теми же компонентами $(\varrho U)$, но для которого $u_{2-}<u_{2,-}^{c r}$, отвечающего верхнему критическому решению. 
Если же выполнены условия (15), (16) и

$$
\frac{4[P]}{c_{0}[\varrho]}-\omega^{2}>0
$$

для критического значения управления

$$
u_{2,-}^{c r}=\omega / 2
$$

существует однофронтовое монотонно убывающее стабилизирующееся решение системы $(8)$, для которого левое предельное значение $\left(\varrho_{-}^{c r}, U_{-}^{c r}, u_{2,-}^{c r}\right) \in \Sigma_{+}$ и

$$
u_{2,+}^{c r}=\frac{1}{2}\left(\omega-\sqrt{\frac{|[\varrho]|}{\varrho+}\left(\frac{4[P]}{c_{0}[\varrho]}-\omega^{2}\right)}\right) .
$$

Более того, не существует монотонно убывающее стабилизирующееся решение системы $(8)$ с теми же компонентами $\varrho U$, но для которого $u_{2-}>u_{2,-}^{c r}$, отвечающего нижнему критическому решению. Отметим, что условие (17) справедливо, если

$$
0<U_{-}<\sqrt{\frac{2 \varrho_{+}}{\varrho_{-}}}\left(-1+\sqrt{\frac{4 \varrho-}{c_{0} \varrho_{+}}}\right) \sqrt{\frac{[P]}{[\varrho]}}
$$

6.6. О бифуркации верхнего критического решения. Теперь получим бифуркацию типа горбатого кинка как возмущение верхнего критического решения, существующего при условиях

$$
\omega^{2}-\frac{4 c_{s}^{2}}{c_{0}}>0, \quad \omega>\omega_{1}>0 .
$$

В чем природа такой бифуркации? Как мы показали выше, верхнее критическое решение, которое мы получаем в фазовой плоскости подходом сверху к критическому многообразию $\Sigma_{+}$, если ось $u_{2}$ направлена вертикально вверх, есть предел существования классической однофронтовой ударной волны. Попытка опуститься ниже критического многообразия приводит к распаду классической ударной волны, однофронтовой, на две - замедление критического решения и со старой скоростью сброс предвестника с немонотонным отрицательным профилем типа горбатого кинка, т. е. мы понижаем правое предельное значение $u_{2,+}$, опуская его ниже критического $u_{2,+}^{c r}$ (переход каустики). Таким образом, происходит разрядка критической (неустойчивой) ситуации посредством двухфронтовых ударных волн. Как видим, это есть реконструкция [18, с. 472-473]: «Произвольное возмущение течения складывается из энтропийно-вихревой волны и звуковой волны [...]. Возмущение в целом представляется линейной комбинацией возмущений обоих типов [. . . ].»

После подстановки вида двухфронтового решения (10) в систему ОДУ (8) для

$$
u_{2}=a_{0}\left(\frac{x-x^{*}(t)}{\varepsilon}\right)+b_{0}\left(\frac{x-x_{1}(t)}{\varepsilon}\right)
$$


получаем третье уравнение системы ОДУ двухфронтовой задачи Римана:

$$
\begin{aligned}
\left(a_{0}+\right. & \left.\dot{b}_{0}\right)=-\omega c_{0}\left(\varrho a_{0}-\varrho_{-} a_{0}^{-}\right)-\omega_{1} c_{0}\left(\varrho b_{0}-\varrho_{-} b_{0}^{-}\right)+ \\
& +\left(\omega_{1}-\omega\right) c_{0} \int_{-\infty}^{\tau} \dot{\varrho} b_{0} d s+c_{0}\left(\varrho\left(a_{0}+b_{0}\right)^{2}-\varrho\left(a_{0}^{-}+b_{0}^{-}\right)^{2}+P-\dot{P}^{-}\right) .
\end{aligned}
$$

Потребуем выполнения условия

$$
\omega^{2}>\frac{4[P]}{c_{0}[\varrho]}
$$

существования верхнего критического решения. Разложение (18) будем искать как возмущение верхнего критического решения. Для этого положим

$$
b_{0}(x, t)=u_{2}^{c r}\left(\frac{x-x_{1}(t)}{\varepsilon}\right)
$$

где $\dot{x}_{1}=\omega_{1}$, тем самым ускоряя или замедляя компоненту $u_{2}^{c r}(\tau)$ верхнего критического решения ОДУ $(8)$, отвечающего скорости фронта $\omega$. Предельные константы верхнего критического решения:

$$
u_{2,-}^{c r}=\frac{1}{2}\left(\omega+\sqrt{\frac{|[\varrho]|}{\varrho}\left(\omega^{2}-\frac{4 c_{s}^{2}}{c_{0}}\right)}\right), \quad u_{2,+}^{c r}=\omega / 2 .
$$

Тогда для возмущения $a_{0}$ получим уравнение

$$
\begin{aligned}
\dot{a}_{0}=c_{0} \varrho\left(a_{0}+2 u_{2}^{c r}-\omega\right) a_{0}-c_{0}\left(\omega_{1}-\omega\right)\left(\varrho u_{2}^{c r}-\right. & \left.\varrho-u_{2,-}^{c r}\right)+ \\
& +c_{0}\left(\omega_{1}-\omega\right) \int_{-\infty}^{\tau} \varrho u_{2}^{c r} d s
\end{aligned}
$$

с условиями стабилизации

$$
a_{0}^{-}=0, \quad\left(\dot{a}_{0}\right)^{-}=\left(\dot{a}_{0}\right)^{-}=0 .
$$

В рассматриваемом случае третье условие Гюгонио двухфронтовой задачи запишется в виде

$$
\varrho_{+}\left(a_{0}^{+}-\omega\right) a^{+}-\frac{1}{2}\left(\omega-\omega_{1}\right) \varrho_{ \pm} \sqrt{\frac{|[\varrho]|}{\varrho}\left(\omega^{2}-\frac{4 c_{s}^{2}}{c_{0}}\right)}=0
$$

где плюс или минус в (22) выбирается в зависимости от знака $\omega_{1}-\omega$. Здесь мы использовали справедливость соотношения

$$
-\omega\left[\varrho u_{2}^{c r}\right]+\left[\varrho\left(u_{2}^{c r}\right)^{2}\right]+\frac{1}{c_{0}}[P]=0
$$

для критического управлении $u_{2}^{c r}$. Прежде всего определимся со знаком $\omega_{1}-\omega$. Исследование нулевых изоклин уравнения (21)

$$
a_{ \pm}=\frac{1}{2}\left(-\left(2 u_{2}^{c r}-\omega\right) \pm \sqrt{D_{a}}\right), \quad a_{-}^{-}=\sqrt{\frac{|[\varrho]|}{\varrho_{-}}\left(\omega^{2}-\frac{4 c_{s}^{2}}{c_{0}}\right)}, \quad a_{-}^{+}=0
$$


показывает, что для дискриминанта

$$
D_{a}=\left(2 u_{2}^{c r}-\omega\right)^{2}-\frac{4\left(\omega_{1}-\omega\right)}{\varrho} \int_{\infty}^{\tau} \dot{\varrho} u_{2}^{c r} d s+4\left(\omega_{1}-\omega\right)\left(u_{2}^{c r}-\frac{\varrho_{-}}{\varrho} u_{2,-}^{c r}\right)
$$

выполняется

$$
\begin{gathered}
D_{a}^{-}=\frac{|[\varrho]|}{\varrho_{-}}\left(\omega^{2}-\frac{4 c_{s}^{2}}{c_{0}}\right), \\
D_{a}^{+}=-\frac{4\left(\omega_{1}-\omega\right)}{\varrho^{+}}\left(\varrho_{-} u_{2,-}^{c r}-\varrho_{+} u_{2,+}^{c r}+\int_{-\infty}^{\infty} \dot{\varrho} u_{2}^{c r} d s\right)>0 \quad\left(\text { или } D_{a}^{+}=0\right),
\end{gathered}
$$

если

$$
Z=\omega-\omega_{1}>0
$$

Таким образом, мы замедляем верхнее критическое решение и возмущение $a_{0}$ предвестника. Тогда получаем следующую спектральную задачу по параметру $Z$ :

$$
\begin{gathered}
\dot{a}_{0}=c_{0} \varrho\left(a_{0}+2 u_{2}^{c r}-\omega\right) a_{0}+c_{0} Z\left(\varrho u_{2}^{c r}-\varrho_{-} u_{2,-}^{c r}\right)-c_{0} Z \int_{-\infty}^{\tau} \dot{\varrho}_{2}^{c r} d s \\
a_{0}^{-}=0, \quad\left(\dot{a}_{0}\right)^{-}=\left(\dot{a}_{0}\right)^{+}=0 \\
\left(a_{0}^{+}-\omega\right) a^{+}-\frac{\varrho_{-}}{2 \varrho_{+}} Z \sqrt{\frac{|[\varrho]|}{\varrho_{-}}\left(\omega^{2}-\frac{4 c_{s}^{2}}{c_{0}}\right)}=0 .
\end{gathered}
$$

Задача состоит в нахождении стабилизирующегося решения уравнения (23). Немонотонное стабилизирующееся решение этой задачи назовем одногорбым кинком, если его график $y=a_{-}$стартует из нуля при $\tau=-\infty$ и убывает, находясь между нулевыми изоклинами, до пересечения с нижней изоклиной в точке его минимума. Затем, находясь под нижней изоклиной, возрастает, стабилизируясь к $u_{2,+}^{c r}$ при $\tau \rightarrow+\infty$. Соответственно, двугорбым кинком назовем немонотонное стабилизирующееся решение этой задачи, график $y=a_{-}$которого стартует из нуля при $\tau=-\infty$ и убывает, находясь между нулевыми изоклинами, до пересечения с нижней изоклиной в точке его минимума. Затем, находясь под нижней изоклиной, возрастает до пересечения с ней в точке его максимума. Далее убывает, находясь между нулевыми изоклинами, и стабилизируется к $u_{2,+}^{c r}$ при $\tau \rightarrow+\infty$. В [35] при выполнении условия (16), оценки (20) и

$$
\omega>Z>\sqrt{\frac{|[\varrho]|}{\varrho_{-}}\left(\omega^{2}-\frac{4 c_{s}^{2}}{c_{0}}\right)}
$$

доказано существование немонотонного решения задачи (23) с профилем типа «одногорбого кинка».

6.7. О бифуркации нижнего критического решения. Исследование бифуркации нижнего критического решения аналогично приведенному выше для 
верхнего критического решения. Бифуркация однофронтового решения в интервале скоростей

$$
\frac{4 c_{s}^{2}}{c_{0}}-\omega^{2}>0, \quad \omega>\omega_{1}>0
$$

рассматривается как возмущение нижнего критического однофронтового решения. В чем природа такой бифуркации? Как мы показали выше, нижнее критическое решение, которое мы получаем в фазовой плоскости подходом снизу к критическому многообразию $\Sigma_{+}$, если ось $u_{2}$ направлена вертикально вверх, есть предел существования классической однофронтовой ударной волны. Попытка подняться выше критического многообразия приводит к распаду классической ударной волны, однофронтовой, на две - замедление критической волны и со старой скоростью сброс предвестника. Таким образом, происходит разрядка критической (неустойчивой) ситуации посредством двухфронтовых ударных волн.

7. Критическая волна разрежения [35]. Начнем с волн разрежения в области строгой гиперболичности, когда $\left(\varrho, U, u_{2}\right)$ не принадлежит критическому множеству $\Sigma_{+}$фазового пространства. Из классической теории каждому собственному вектору соответствует волна разрежения. Действительно, будем искать волну разрежения в виде

$$
\varrho=\varrho(x / t), \quad U=U(x / t), \quad u_{2}=u_{2}(x / t) .
$$

Подставляя эти равенства в систему уравнений (7) и обозначая $s=x / t$, а точкой - производную по $s$, получаем

$$
\begin{aligned}
& -s \dot{\varrho}+(\varrho \dot{U})=0, \\
& -s(\varrho \dot{U})+\left(\varrho U^{2}+2 \dot{P}\right)=0, \\
& -s\left(\varrho \dot{u}_{2}\right)+\left(\varrho u_{2}^{2}+\dot{P} / c_{0}\right)=0 .
\end{aligned}
$$

Откуда либо

$$
\left(\begin{array}{ccc}
U-s & \varrho & 0 \\
U^{2}-s U+2 P^{\prime} & 2 \varrho U-s \varrho & 0 \\
u_{2}^{2}-s u_{2}+P^{\prime} / c_{0} & 0 & 2 \varrho u_{2}-s \varrho
\end{array}\right)\left(\begin{array}{c}
\dot{\varrho} \\
\dot{U} \\
\dot{u}_{2}
\end{array}\right)=0
$$

либо

$$
\varrho=\text { const }, \quad U=\text { const }, \quad u_{2}=\text { const, }
$$

либо вектор $\left(\dot{\varrho}, \dot{U}, \dot{u}_{2}\right)^{\mathrm{T}}=R_{\lambda}$ - собственный для матрицы $\mathscr{A}$, а $q=\lambda$, соответствующему собственному значению. В дальнейшем фиксируем $c_{0} \in(0,1)$ и рассмотрим случай $\varrho>0, U>0, u_{2}>0$. Будем считать, что левое состояние находится на критическом многообразии $\left(\varrho_{-}, U_{-}, u_{2,-}\right)^{\mathrm{T}} \in \Sigma_{+}$. Из алгебраического исследования системы $(24)$ следует, что на $\Sigma_{+}$матрица $\mathscr{A}$ имеет два собственных вектора. В конусе $[\varrho]>0,[U]>0,\left[u_{2}\right]>0$, уменьшая значение $u_{2,-}$, мы выйдем на первую точку пересечения прямой $\varrho \equiv \varrho_{-}, U \equiv u_{-}$, с критической гиперповерхностью $\Sigma_{+}$, так что левое состояние $\left(\varrho_{-}, U_{-}, U_{2,-}\right) \in \Sigma_{+}$ и правое состояние $\left(\varrho_{+}, U_{+}, U_{2,+}\right)$ лежат выше $\Sigma_{+}$. Тогда

$$
u_{2,-}^{c r}=\frac{1}{2}\left(U_{-}+\sqrt{2 P^{\prime}\left(\varrho_{-}\right)}\right) .
$$


Для достаточно малого $\left[u_{2}\right]$ существует решение системы $(24)$, определяющее часть волны разрежения между левым $\left(\varrho_{-}, U_{-}, U_{2,-}\right)$ и правым $\left(\varrho_{+}, U_{+}, U_{2,+}\right)$ состояниями. Это критическая волна разрежения, т. е. существуют волны разрежения с левым $\left(\varrho_{-}, U_{-}, U_{2,-}+\varepsilon\right)$ и правым $\left(\varrho_{+}, U_{+}, U_{2,+}\right)$ состояниями для сколь угодно малого $\varepsilon>0$. Таким образом, мы не можем соединить волной разрежения точки фазового пространства, лежащие по разные стороны верхней критической гиперповерхности $\Sigma_{+}$в некоторой малой ее окрестности. Теперь рассмотрим второй случай, когда

$$
0<u_{2}<P^{\prime}(0) / c
$$

В этом случае получим $d \varrho / d m_{2}>0 \forall s \in \mathbb{R}$, если

$$
\frac{2 m_{2}}{\varrho}-\frac{m_{1}}{\varrho}-\sqrt{2 P^{\prime}}<0,
$$

т. е. $\lambda_{1}<\lambda_{3}<\lambda_{2}$ (под верхней гиперповерхностью $\Sigma_{+}$критического многообразия). Здесь $m_{1}=\varrho U, m_{2}=\varrho u_{2}$. В конусе $[\varrho]>0,\left[m_{1}\right]>0,\left[m_{2}\right]>0$, увеличивая значение $u_{2,+}$, мы выйдем на первую точку пересечения прямой $\varrho \equiv \varrho_{+}, U \equiv U_{+}$, с критической гиперповерхностью $\Sigma_{+}$, так что правое состояние $\left(\varrho_{+}, U_{+}, u_{2,+}\right)^{\mathrm{T}} \in \Sigma_{+}$и левое состояние $\left(\varrho_{-}, U_{-}, u_{2,-}\right)^{\mathrm{T}}$ лежат ниже $\Sigma_{+}$. Тогда

$$
u_{2,+}^{c r}=\frac{1}{2}\left(U_{+}+\sqrt{2 P^{\prime}\left(\varrho_{+}\right)}\right) .
$$

Для достаточно малого $\left[u_{2}\right]$ существует решение системы $(24)$, определяющее часть волны разрежения между левым $\left(\varrho_{-}, U_{-}, u_{2,-}\right)^{\mathrm{T}}$ и правым $\left(\varrho_{+}, U_{+}, u_{2,+}\right)^{\mathrm{T}}$ состояниями. Это критическая волна разрежения, т. е. существуют волны разрежения с правым $\left(\varrho_{+}, U_{+}, u_{2,+}-\varepsilon\right)^{\mathrm{T}}$ и левым $\left(\varrho_{-}, U_{-}, u_{2,-}\right)^{\mathrm{T}}$ состояниями для сколь угодно малого $\varepsilon>0$. Таким образом, и в этом случае мы не можем соединить волной разрежения точки фазового пространства, лежащие по разные стороны верхней критической гиперповерхности $\Sigma_{+}$в некоторой малой ее окрестности.

8. Бифуркация верхней критической волны разрежения. Будем считать, что левое состояние находится на критическом многообразии $\left(\varrho_{-}, U_{-}, u_{2,-}\right)^{\mathrm{T}} \in$ $\Sigma_{+}, c=$ const. Положим $s^{ \pm}=\lambda_{2}\left(U^{ \pm}, \varrho^{ \pm}\right)$. Тогда, как мы формулировали выше, существует верхняя критическая волна разрежения. В случае

$$
\omega<s^{-}=U^{-}+\sqrt{2 P^{\prime}\left(\varrho^{-}\right)}
$$

в переменных $\varrho, m_{1}=\varrho U, m_{2}=\varrho u_{2}$ система (24) запишется в виде

$$
\begin{gathered}
-s \dot{\varrho}+\left(m_{1}\right)=0 \\
\left(-s+\frac{2 m_{1}}{\varrho}\right) \dot{m}_{1}+\left(2 P^{\prime}(\varrho)-\frac{m_{1}^{2}}{\varrho^{2}}\right) \dot{\varrho}=0 \\
\left(-s+\frac{2 m_{2}}{\varrho}\right) \dot{m}_{2}+\left(-\frac{m_{2}^{2}}{\varrho^{2}}+\frac{P^{\prime}(\varrho)}{c}\right) \dot{\varrho}=0 .
\end{gathered}
$$

Рассмотрим возмущение верхней критической волны разрежения:

$$
\varrho=\varrho(x / t)+A(1-\theta)(x-\omega t),
$$




$$
\begin{aligned}
& m_{1}=m_{1}(x / t)+B(1-\theta)(x-\omega t), \\
& m_{2}=m_{2}^{c r}(x / t)+\beta(1-\theta)(x-\omega t),
\end{aligned}
$$

где $\left(\varrho(x / t), m_{1}(x / t), m_{2}^{c r}(x / t)\right)^{\mathrm{T}}$ - критическая волна разрежения. Для достаточно малого $[\varrho]>0$ существует возмущение верхней критической волны разрежения, так что

$$
\beta=\varrho_{-}\left(\omega-2 u_{2,-}^{c r}\right)<\varrho_{-}\left(s^{-}-2 u_{2,-}^{c r}\right)<0 .
$$

Доказательство этого факта смотри в [36]. Там же приведено доказательство существования бифуркации нижней критической волны разрежения.

Выводы. Какие можно сделать выводы? Как мы отмечали выше, существующие формы моделирования турбулентности отражают две крайности волновую (вибрационная газовая динамика, см., например, [1]) и наиболее распространенную диффузионную (статистическую) теорию турбулентности $[16,17,21]$. В то же время эксперимент показывает, что турбулентность есть сочетание и взаимодействие двух факторов-волнового и диффузионного. Существование двух типов возмущений с разными скоростями перемещения по газу (звуковые - со скоростью звука и энтропийно-вихревые - со скоростью потока газа) позволяет выдвинуть гипотезу существования на мезоструктурном уровне двухскоростной гидродинамики, когда разные части газа обладают разными скоростями относительно неподвижного газа, разными коэффициентами переноса, если рассматривать поток этих флуктуаций, обусловленный градиентом их плотностей (точнее, химическим потенциалом). То, что энтропийно-вихревые возмущения могут рассматриваться как флуктуации плотности, может быть обосновано соответствующим законом сохранения - законом сохранения циркуляции скорости (теорема Томпсона). Очевидно, что это волновые характеристики процесса. В основе классической гидродинамики лежит система Эйлера, регуляризованная за счет моделирования вязкости и турбулентости. Как мы показали, переход при больших числах Рейнольдса от односкоростной модели к двухскоростной связан с потерей строгой гиперболичности системы Эйлера. Двухскоростная модель возникает по той причине, что в общем положении потеря строгой гиперболичности связана с появлением двукратных корней дисперсионного уравнения. Все полученные эффекты есть внутренние свойства самой системы Эйлера, связанные со структурой собственного пространства на критических многообразиях (появление присоединенных векторов). Построенные выше бифуркации критических ударных волн дают ответ на давно поставленную Лаксом задачу о том, что происходит с ударной волной при прохождении ею в фазовом пространстве критического многообразия. Процесс остается волновым. Попытка опуститься ниже критического многообразия приводит к распаду классической ударной волны, однофронтовой, на две - замедление верхнего критического решения и со старой скоростью сброс предвестника с немонотонным отрицательным профилем. Таким образом, происходит разрядка критической (неустойчивой) ситуации посредством двухфронтовых ударных волн.

Теперь отметим (см. [21]), что в газе наряду с падающей звуковой волной мы еще имеем и отраженную звуковую и энтропийно-вихревую волны, а на самой поверхности разрыва возникает рябь (аналог волны Марангони 
(см. [12]). Согласуясь с результатами [21], можно утверждать, что в двумерном случае бифуркация критической устойчивой ударной волны приведет к перемежаемости (полостчатой структуре), затем на самой поверхности разрыва возникнет рябь аналога волны Марангони (см. [12]), которая породит срывающиеся с фронта ударной волны струи (аналог дендритов в кристаллизации (см. [12]). Как видим, это уже факторы сочетания волновых и диффузионных свойств процесса. Можно выдвинуть гипотезу, что в двумерном случае бифуркация критической волны разрежения [19] приведет к образованию вихрей. Оба эти утверждения имеют отношение к реконструкции пункта 6) базовых свойств внутренней турбулентности. Проведенные исследования дают надежду, что природа построенных бифуркаций имеет общий характер.

\section{ORCID}

Евгений Владимирович Радкевич: http://orcid.org/0000-0001-7904-4476

Владимир Владимирович Палин: http://orcid.org/0000-0001-7529-7855

\section{БИБЛИОГРАФИЧЕСКИЙ СПИСОК}

1. Раушенбах Б. В. Вибрационное горение. М.: Физматлит, 1961. 500 с.

2. Колмогоров А. Н. Локальная структура турбулентности в несжимаемой вязкой жидкости при очень больших числах Рейнольдса // Докл. АН СССР, 1941. Т. 30, № 4. С. 299303; Колмогоров А. Н. Локальная структура турбулентности в несжимаемой вязкой жидкости при очень больших числах Рейнольдса // УФН, 1967. Т. 93, № 11. С. 476-481, http://ufn.ru/ru/articles/1967/11/h/.

3. Prandtl L. Über die ausgebildete Turbulenz / Verhandlungen Kongreß Zürich, 1927. pp. 6274, SBAWW_137_2a_0307-0325.pdf ; Prandtl L. Über die ausgebildete Turbulenz / Ludwig Prandtl Gesammelte Abhandlungen; eds. Walter Tollmien, Hermann Schlichting, Henry Görtler, F. W. Riegels, 1961. pp. 736-751. doi: 10.1007/978-3-662-11836-8_60.

4. Лойцянский Л. Г. Механика жидкости и газа, 6-е изд.. М.: Наука, 1987.

5. Clauser F. H. The Turbulent Boundary Layer // Advances in Applied Mechanics, 1956. vol. 4. pp. 1-51. doi : 10.1016/s0065-2156(08)70370-3.

6. Лукашев Е. А., Палин В. В., Радкевич Е. В. Яковлев Н. Н. О неклассической регуляризации многокомпонентной системы Эйлера // Пробл. мат. анал., 2013. №73. С. 67-86.

7. Радкевич Е. В. О природе бифуркаций однофронтовых решений усеченной системы Эйлера // Пробл. мат. анал., 2013. №73. С. 125-139.

8. Каблов Е. Н. С. Т. Кишкин и его вклад в развитие науки и материаловедения / Труды международной научно-технической конференции, посвященной 100-летию со дня рождения академика С. Т. Кишкина. М.: ВИАМ, 2006. С. 5-11.

9. Cahn J. W., Hilliard J. E. Free Energy of a Nonuniform System. I. Interfacial Free Energy // J. Chem. Phys., 1958. vol.28, no.2. pp. 258-267. doi:10.1063/1.1744102 ; Cahn J. W., Hilliard J. E. Free Energy of a Nonuniform System. I. Interfacial Free Energy / The Selected Works of John W. Cahn; eds. W. Craig Carter, William C. Johnson. pp. 29-38. doi: 10. 1002/9781118788295.ch4.

10. Cahn J. W., Hilliard J. E. Free Energy of a Nonuniform System. III. Nucleation in a Two-Component Incompressible Fluid// J. Chem. Phys., 1959. vol.31, no.3. pp. 688699. doi: 10.1063/1.1730447 ; Cahn J. W., Hilliard J. E. Free Energy of a Nonuniform System. III. Nucleation in a Two-Component Incompressible Fluid / The Selected Works of John W. Cahn; eds. W. Craig Carter, William C. Johnson. pp. 39-50. doi: 10.1002/ 9781118788295. ch5.

11. Яковлев Н. Н., Лукашев Е. А., Радкевич Е. В. Проблемы реконструкции процесса направленной кристаллизации // Докл. РАН, 2008. Т. 421, № 5. С. 625-629.

12. Lukashev E. A., Radkevich E. V. Solidification and Structuresation of Instability Zones // Applied Mathematics, 2010. vol.1. pp. 159-178. doi : 10.4236/am.2010.13021. 
13. Яковлев Н. Н., Лукашев Е. А., Радкевич Е. В. Исследование процесса направленной кристаллизации методом математической реконструкции // Докл. РАН, 2012. Т. 445, № 4. C. 398-401.

14. Лапин Ю. В. Статистическая теория турбулентности (прошлое и настоящее-краткий очерк идей) // Научно технические ведомости СПбГТУ, 2004. № 2, Google Scholar Cluster: 796228169334250115.

15. Bradshaw P. An Introduction to Turbulence and its Measurement/ The Commonwealth and International Library. Thermodynamics and Fluid Mechanics Division/ ed. W. A. Woods. Oxford etc.: Pergamon Press, 1971. xviii+218 pp.

16. Монин А. С., Яглом А. М. Статистическая гидромеханика. Т. 1: Механика турбулентности. М.: Наука, 1965. 640 с.

17. Modern Developments in Fluid Dynamics: An Account of Theory and Experiment Relating to Boundary Layers, Turbulent Motion and Wakes: Composed by the Fluid Motion Panel of the Aeronautical Research Committee and other/ Oxford Engineering Science Series. T. 1, 2 / ред. Sydney Goldstein. Oxford: Clarendon Press, 1938; Современное состояние гидроаэродинамики вязкой жидкости. Т. 1, 2/ ред. С. Гольдштейн. М.: Иностр. лит., 1948.

18. Ландау Л. Д., Лифшиц Е. М. Теоретическая физика. Т. 6: Гидродинамика, 3-е изд.. М.: Наука, 1986. 736 c.

19. Nicolis G., Prigogine I. Self-Organization in Nonequilibrium Systems. From Dissipative Structures to Order through Fluctuations. New York etc.: J. Wiley \& Sons, 1977. 491 c.; Николис Г., Пригожин И. Самоорганизация в неравновесных системах (от dиссипативных структур к упорядоченности через флуктуации). М.: Мир, 1979. 512 с.

20. Sheih C. M. Airborne Hot-Wire Measurements of the Small-Scale Structure of Atmospheric Turbulence // Phys. Fluids, 1971. vol. 14, no. 2. pp. 201-215. doi: 10.1063/1.1693416.

21. Кузнецов В. Р., Сабельников В. А. Турбулентность и горение. М.: Наука, 1986. 287 с.

22. Corrsin S. Investigation of the flow field of a highly heated jet of air: NACA W-94 ACR No. 3123, 1943.

23. Лукашев Е. А., Радкевич Е. В., Яковлев Н. Н. О визуализации начальной стадии кристаллизации бинарных сплавов // Наноструктуры. Математическая физика и моделирование, 2014. Т. 11, № 2. С. 5-36.

24. Рахматулин Х. А. Основы газодинамики взаимопроникающих движений сжимаемых сред // ПММ, 1956. Т. 20, № 2. С. 184-195.

25. Нигматулин Р. И. Динамика многофазных сред. М.: Наука, 1987.

26. Dreyer W., Wagner B. Sharp-Interface Model for Eutectic Alloys. Part I. Concentration Dependent Surface Tension// Interfaces and Free Boundaries, 2005. vol.7, no. 2. pp. 199-227. doi: 10.4171/ifb/121.

27. Радкевич Е. В. Математические вопросы неравновесных прочессов/ Белая серия в математической физике. Т. 4. Новосибирск: Рожковская, 2007. 300 с.

28. Wohl K, Shore L., von Rosenberg H., Weil C. W. The burning velocity of turbulent flames // Symposium (International) on Combustion, 1953. T. 4, № 1 (Fourth Symposium (International) on Combustion). C. 620-635. doi: 10.1016/S0082-0784(53)80083-4; Воль К., Шор Л., Розенберг Х., Уейл С. Скорость распространения турбулентного пламени / Вопросы горения и детонационных волн: Четвертый международный симпозиум по вопросам горения и детонационных волн. М.: Оборониздат, 1958. С. 425-439.

29. Карпов В. П., Соколик А. С.// Докл. АН СССР, 1961. Т. 138. С. 874.

30. Франк-Каменецкий Д. А. Диффузия и mеплопередача в химической кинетике. М.: Наука, 1967. 502 с.

31. Кузнецов В. Р. Скорость распространения пламени в турбулентном потоке гомогенной горючей смеси / Горение и взрыв: Материалы 4-го всесоюзного симпозиума по горению и взрыву (23-27 сентября 1974 г.; Ленинград); ред. Л. Н. Стесик. М.: Наука, 1977. C. $366-372$. 
32. Wohl K., Shore L. Experiments with Butane-Air and Methane-Air Flames // Ind. Eng. Chem., 1955. vol. 47, no. 4. pp. 828-834. doi: 10.1021/ie50544a047.

33. Рыков Ю. Г., Лысов В. Г., Феодоритова О. Б. Возникновение неклассических скачков в одной модели течения двухкомпонентной, двухскоростной сплошной среды: Препринт ИПМ им. М. В. Келдыша, № 74, 2012. 20 с.

34. Majda A., Pego R. L. Stable viscosity matrices for systems of conservation laws // J. Differ. Equ., 1985. vol.56, no. 2. pp. 229-262. doi : 10.1016/0022-0396 (85)90107-x.

35. Lax Peter D. Hyperbolic Partial Differential Equations / Courant Lecture Notes, 14. New York: New York University, Courant Institute, 2006. 217 pp.

36. Палин В. В., Радкевич Е. В. О бифуркации критических волн разрежения // Пробл. мат. анал., 2014. №76. С. 121-132.

Поступила в редакцию 20/XII/2014;

в окончательном варианте - 05/II/2015;

принята в печать $-25 / \mathrm{II} / 2015$.

Vestn. Samar. Gos. Techn. Un-ta. Ser. Fiz.-mat. nauki

[J. Samara State Tech. Univ., Ser. Phys. \& Math. Sci.], 2015, vol. 19, no. 1, pp. 155-185

ISSN: 2310-7081 (online), 1991-8615 (print)

doi: http://dx.doi.org/10.14498/vsgtu1418

MSC: 35Q31, 35B32

\title{
ON THE INNER TURBULENCE PARADIGM
}

\author{
N. N. Yakovlev ${ }^{1}$, E. A. Lukashev ${ }^{1}$, \\ E. V. Radkevich ${ }^{2}$, V. V. Palin ${ }^{2}$ \\ 1 Joint-stock company Turaevo Machine-Building Design Bureau "SOYUZ", \\ 10, Turaevo st., Lytkarino, 140080, Russian Federation. \\ 2 M. V. Lomonosov Moscow State University, \\ Faculty of Mechanics and Mathematics, \\ Vorob'evy gory, Moscow, 119899, Russian Federation.
}

\begin{abstract}
In the paper we study the reproducing of the initial phase of the inner turbulence (without regard for the boundary effects). The atypical regularization of multiple-component Euler system is made by the viscosity and diffuse layering introduction. The analogue of Hugoniot condition and the analogue of Lax stability condition are constructed for it. The problem of local accessibility of the phase space points is investigated. The bifurcations of one-front solutions of the abridged Euler system to the two-front solutions are obtained. The supersonic behaviour of bifurcations appearance is shown. The
\end{abstract}

(C) 2015 Samara State Technical University.

How to cite Reference

Yakovlev N. N., Lukashev E. A., Radkevi ch E. V., P alin V. V. On the inner turbulence paradigm, Vestn. Samar. Gos. Tekhn. Univ., Ser. Fiz.-Mat. Nauki [J. Samara State Tech. Univ., Ser. Phys. \& Math. Sci.], 2015, vol. 19, no. 1, pp. 155-185. doi: 10.14498/vsgtu1418. (In Russian)

\section{Authors Details}

Nikolay N. Yakovlev ( Cand. Phys. \& Math. Sci.; amntksoyuz@mail.ru), General Manager.

Evgeniy A. Lukashev (Dr. Techn. Sci.; elukashov@yandex.ru), Chief Specialist.

Evgeniy V. Radkevich (Dr. Phys. \& Math. Sci.; evrad07@gmail.com; Corresponding Author), Professor, Dept. of Differential Equiations.

Vladimir V. Palin (Cand. Phys. \& Math. Sci.; grey_stranger84@mail.ru), Assistant, Dept. of Differential Equiations. 
reconstruction of the initial phase of the inner turbulence (without regard for the boundary effects) is made including the mathematical description of the birth of two-speed flow (the Riemann-Hugoniot catastrophe) and alternation.

Keywords: inner turbulence reconstruction, two-speed flow, the RiemannHugoniot catastrophe, alternation, bifurcation, Euler system, kinetic equation.

doi: http://dx.doi.org/10.14498/vsgtu1418

\section{ORCID}

Evgeniy V. Radkevich: http://orcid.org/0000-0001-7904-4476

Vladimir V. Palin: http://orcid.org/0000-0001-7529-7855

\section{REFERENCES}

1. Raushenbakh B. V. Vibratsionnoe gorenie [Vibration combustion]. Moscow, Fizmatlit, 1961, 500 pp. (In Russian)

2. Kolmogorov A. N. Local structure of turbulence in an incompressible viscous fluid at very high Reynolds numbers, Sov. Phys. Usp., 1968, vol. 10, no.6, pp. 734-746. doi: 10.1070/ PU1968v010n06ABEH003710.

3. Prandtl L. Über die ausgebildete Turbulenz, Verhandlungen Kongreß Zürich, 1927, pp. 6274, SBAWW_137_2a_0307-0325.pdf ; Prandtl L. Über die ausgebildete Turbulenz, Ludwig Prandtl Gesammelte Abhandlungen; eds. Walter Tollmien, Hermann Schlichting, Henry Görtler, F. W. Riegels, 1961, pp. 736-751. doi : 10.1007/978-3-662-11836-8_60.

4. Loytsyanskiy L. G. Mekhanika zhidkosti i gaza [Fluid Mechanics]. Moscow, Nauka, 1987 (In Russian).

5. Clauser F. H. The Turbulent Boundary Layer, Advances in Applied Mechanics, 1956, vol. 4, pp. 1-51. doi: 10.1016/s0065-2156(08)70370-3.

6. Lukashev E. A., Palin V. V., Radkevich E. V., Yakovlev N. N. Nonclassical Regularization of the Multicomponent Euler System, Journal of Mathematical Sciences, 2014, vol. 196, no. 3, pp. 322-345. doi : 10.1007/s10958-014-1661-1.

7. Radkevich E. V. On the Nature of Bifurcations of One-Front Solutions of the Truncated Euler System, Journal of Mathematical Sciences, 2014, vol. 196, no. 3, pp. 388-404. doi : 10. 1007/s10958-014-1664-y.

8. Kablov E. N. S. T. Kishkin and its contribution to development of science and materials science, Trudy mezhdunarodnoi nauchno-tekhnicheskoi konferentsii, posviashchennoi 100letiiu so dnia rozhdeniia akademika S. T. Kishkina [Proceedings of the International scientific conference dedicated to the 100th anniversary of academician S. T. Kishkin]. Moscow, VIAM, 2006, pp. 5-11 (In Russian).

9. Cahn J. W., Hilliard J. E. Free Energy of a Nonuniform System. I. Interfacial Free Energy, J. Chem. Phys., 1958, vol.28, no.2, pp. 258-267. doi: 10.1063/1.1744102 ; Cahn J. W., Hilliard J. E. Free Energy of a Nonuniform System. I. Interfacial Free Energy, The Selected Works of John W. Cahn; eds. W. Craig Carter, William C. Johnson, pp. 29-38. doi: 10. 1002/9781118788295.ch4.

10. Cahn J. W., Hilliard J. E. Free Energy of a Nonuniform System. III. Nucleation in a Two?Component Incompressible Fluid, J. Chem. Phys., 1959, vol.31, no.3, pp. 688699. doi: 10.1063/1.1730447 ; Cahn J. W., Hilliard J. E. Free Energy of a Nonuniform System. III. Nucleation in a Two?Component Incompressible Fluid, The Selected Works of John W. Cahn; eds. W. Craig Carter, William C. Johnson, pp. 39-50. doi: 10.1002/ $9781118788295 . \operatorname{ch} 5$.

11. Yakovlev N. N., Lukashev E. A., Radkevich E. V. Problems of reconstruction of the process of directional solidification, Dokl. Phys., 2008, vol.53, no.8, pp. 442-446. doi: 10.1134/ S1028335808080090. 
12. Lukashev E. A., Radkevich E. V. Solidification and Structuresation of Instability Zones, Applied Mathematics, 2010, vol.1, pp. 159-178. doi: 10.4236/am.2010.13021.

13. Yakovlev N. N., Lukashev E. A., Radkevich E. V. Investigation of directed crystallization by the method of mathematical reconstruction, Dokl. Phys., 2012, vol.57, no. 8, pp. 297-300. doi : 10.1134/S1028335812080046.

14. Lapin Yu. V. Statistical theory of turbulence. Past, and Present. A brief outline of ideas, Nauchno tekhnicheskie vedomosti SPbGTU, 2004, no. 2 (In Russian), Google Scholar Cluster: 796228169334250115.

15. Bradshaw P. An Introduction to Turbulence and its Measurement, The Commonwealth and International Library. Thermodynamics and Fluid Mechanics Division, ed. W. A. Woods. Oxford etc., Pergamon Press, 1971, xviii+218 pp.

16. Monin A. S., Yaglom A. M. Statistical Fluid Mechanics, vol. 1, Mechanics of Turbulence, ed. John L. Lumley. Cambridge, Mass., M.I.T. Press, 1971, xii+770 pp.

17. Modern Developments in Fluid Dynamics: An Account of Theory and Experiment Relating to Boundary Layers, Turbulent Motion and Wakes, Composed by the Fluid Motion Panel of the Aeronautical Research Committee and other, Oxford Engineering Science Series, vol. 1, 2, ed. Sydney Goldstein. Oxford, Clarendon Press, 1938.

18. Landau L. D., Lifshitz E. M. A Course of Theoretical Physics, vol.6, Fluid Mechanics. Oxford etc., Pergamon Press, 1959, xiii+539 pp.

19. Nicolis G., Prigogine I. Self-Organization in Nonequilibrium Systems. From Dissipative Structures to Order through Fluctuations. New York etc., J. Wiley \& Sons, 1977, 491 pp.

20. Sheih C. M. Airborne Hot-Wire Measurements of the Small-Scale Structure of Atmospheric Turbulence, Phys. Fluids, 1971, vol. 14, no. 2, pp. 201-215. doi: 10.1063/1.1693416.

21. Kuznetsov V. R., Sabel'nikov V. A. Turbulentnost' i gorenie [Turbulence and Combustion]. Moscow, Nauka, 1986, 287 pp. (In Russian)

22. Corrsin S. Investigation of the flow field of a highly heated jet of air, NACA W-94 ACR No. 3123, 1943.

23. Lukashev E. A., Radkevich E. V., Yakovlev N. N. On the visualization of the initial stage of crystallization of binary alloys, Nanostruktury. Matematicheskaia fizika $i$ modelirovanie, 2014, vol. 11, no. 2, pp. 5-36 (In Russian).

24. Rakhmatulin Kh. A. The fundamentals of gas dynamics of interpenetrating motions of compressible media, Prikladnaia Matematika i Mekhanika, 1956, vol. 20, no. 2, pp. 184-195 (In Russian).

25. Nigmatulin R. I. Dinamika mnogofaznykh sred [Dynamics of Multiphase Media]. Moscow, Nauka, 1987 (In Russian).

26. Dreyer W., Wagner B. Sharp-Interface Model for Eutectic Alloys. Part I. Concentration Dependent Surface Tension, Interfaces and Free Boundaries, 2005, vol. 7, no. 2, pp. 199-227. doi: $10.4171 /$ ifb/121.

27. Radkevich E. V. Matematicheskie voprosy neravnovesnykh protsessov [Mathematical problems of nonequilibrium processes], Belaia seriia v matematicheskoi fizike [White Series in Mathematical Physics], vol.4. Novosibirsk, Rozhkovskaia, 2007, 300 pp. (In Russian)

28. Wohl K, Shore L., von Rosenberg H., Weil C. W. The burning velocity of turbulent flames, Symposium (International) on Combustion, 1953, vol. 4, no. 1 (Fourth Symposium (International) on Combustion), pp. 620-635. doi: 10.1016/S0082-0784(53)80083-4.

29. Karpov V. P., Sokolik A. S., Dokl. AN SSSR, 1961, vol. 138, pp. 874 (In Russian).

30. Frank-Kamenetskii D. A. Diffusion and heat transfer in chemical kinetics. New York, Plenum Press, 1969, 574 pp.

31. Kuznetsov V. R. The flame propagation velocity in the turbulent flow of the homogeneous combustible mixture, Gorenie $i$ vzryv [Burning and explosion]; ed. L. N. Stesik. Moscow, Nauka, 1977, pp. 366-372 (In Russian).

32. Wohl K., Shore L. Experiments with Butane-Air and Methane-Air Flames, Ind. Eng. Chem., 1955, vol. 47, no. 4, pp. 828-834. doi: 10.1021/ie50544a047. 
33. Rykov Yu. G., Lysov V. G., Feodoritova O. B. Appearing nonclassical jumps in a model of a flow of two-component two-velocity medium, Preprint of the Keldysh Institute of Applied Mathematics of RAS, No. 74, 2012, 20 pp. (In Russian)

34. Majda A., Pego R. L. Stable viscosity matrices for systems of conservation laws, J. Differ. Equ., 1985, vol. 56, no. 2, pp. 229-262. doi: 10.1016/0022-0396(85) 90107-x.

35. Lax Peter D. Hyperbolic Partial Differential Equations, Courant Lecture Notes, 14. New York, New York University, Courant Institute, 2006, 217 pp.

36. Palin V. V., Radkevich E. V. Bifurcations of Critical Rarefaction Waves, Journal of Mathematical Sciences, 2014, vol.202, no.2, pp. 245-258. doi:10.1007/ s10958-014-2044-3.

Received 20/XII/2014;

received in revised form $05 / \mathrm{II} / 2015$;

accepted 25/II/2015. 